\title{
Multistep loading of PCNA onto DNA by RFC
}

\author{
Marina Schrecker ${ }^{1 *}$, Juan C. Castaneda ${ }^{2,3 *}$, Sujan Devbhandari², Charanya Kumar ${ }^{2}$, Dirk \\ Remus²\# and Richard K. Hite ${ }^{1} \#$
}

${ }^{1}$ Structural Biology Program, Memorial Sloan Kettering Cancer Center; New York, New York 10065, USA

${ }^{2}$ Molecular Biology Program, Memorial Sloan Kettering Cancer Center

${ }^{3}$ Weill Cornell Medicine Graduate School, Weill Cornell Medicine; New York, New York 10065, USA

*These authors contributed equally

\#Corresponding author. Email: hiter@mskcc.org, remusd@mskcc.org 


\section{Abstract:}

The DNA sliding clamp proliferating cell nuclear antigen (PCNA) is an essential cofactor for many eukaryotic DNA metabolic enzymes. PCNA is loaded around DNA by the ATPdependent clamp loader replication factor C (RFC), which acts at single-stranded/doublestranded DNA junctions harboring a recessed 3' end (3' ss/dsDNA junctions) and at DNA nicks. To illuminate the loading mechanism we have investigated the structure of RFC:PCNA bound to ATP $\gamma$ S and 3' ss/dsDNA junctions or nicked DNA using cryogenic electron microscopy. Unexpectedly, we observe open and closed PCNA conformations in the RFC:PCNA:DNA complex, revealing that PCNA can adopt an open, planar conformation that allows direct insertion of dsDNA, and indicating that PCNA ring-closure is not mechanistically coupled to ATP-hydrolysis. By resolving multiple DNA-bound states of RFC:PCNA we observe that partial melting facilitates lateral insertion into the central channel formed by RFC:PCNA. We also resolve the Rfc1 $\mathrm{N}$-terminal domain and demonstrate that its single BRCT domain participates in coordinating DNA prior to insertion into the central RFC channel, which promotes PCNA loading on the lagging strand of replication forks in vitro. Combined, our data suggest a comprehensive and fundamentally revised model for the RFC-catalyzed loading of PCNA onto DNA. 


\section{Introduction:}

Proliferating cell nuclear antigen (PCNA) is an essential co-factor in DNA metabolic processes critical for the maintenance of eukaryotic chromosomes. Initially characterized as a processivity factor for replicative DNA polymerases, PCNA has since been shown to be an interaction hub for a wide array of proteins involved in DNA replication, the repair or bypass of DNA damage, chromatin assembly, chromosome cohesion, and cell cycle regulation ${ }^{1}$. Integral to PCNA function is its ability to topologically encircle DNA and act as a sliding clamp. PCNA forms homotrimeric complexes in which the subunits are arranged head-to-tail to form a closed ring ${ }^{2,3}$. The outer ring surface is shaped by globular $\beta$-sheet domains and harbors the protein interaction sites, while the ring interior is lined with $\alpha$-helices featuring positively charged surface residues that can engage in electrostatic interactions with the DNA phosphate backbone. With an inner diameter of $\sim 34 \AA$ the PCNA ring can comfortably accommodate Bform DNA ( 20 A diameter), facilitating the ability of PCNA to slide along DNA 4 .

Importantly, PCNA also forms stable rings free in solution, preventing its spontaneous binding to DNA in the absence of free DNA ends ${ }^{5-7}$. Consequently, PCNA loading onto DNA is facilitated by clamp loader complexes belonging to the AAA+ family of ATPases that open the PCNA ring and close it around DNA ${ }^{8}$. Two related clamp loaders, RFC and Ctf18-RFC can load PCNA onto DNA. RFC is essential for cell growth and considered the canonical PCNA loader, while Ctf18-RFC is non-essential and appears to perform more specialized roles in replication checkpoint signaling and chromosome cohesion ${ }^{9}$. The DNA substrate specificity of clamp loaders is critical to prevent random and futile clamp loading along chromosome arms and target clamps to relevant sites instead. Accordingly, RFC loads PCNA specifically at single-stranded/double-stranded DNA junctions harboring a recessed 3' end (3' junctions) or at DNA nicks, DNA structures that are commonly formed at sites of DNA replication and repair ${ }^{10-}$ 
15. However, how RFC recognizes 3' junctions, opens the PCNA ring, and inserts DNA into the PCNA ring remains unclear due to a lack of structural information on the RFC:PCNA:DNA complex.

The overall structural organization of RFC has been revealed by X-ray crystallography and cryo-EM analyses of the yeast and human complexes, respectively ${ }^{16,17}$. RFC is composed of four small AAA+ subunits, Rfc2-5, and one large AAA+ subunit, Rfc1, which assemble headto-tail into a two-tiered partially open ring structure. The $\mathrm{N}$-terminal tier contains the AAA+ domains and the unique $A^{\prime}$ domain of Rfc1 (note: yeast nomenclature is used throughout this paper). The C-terminal tier, in contrast, forms a rigid collar composed of $\alpha$-helical domains provided by Rfc1-5. As is characteristic for AAA+ ATPases, ATP is bound at RFC subunit interfaces with catalytic residues at each ATPase site contributed by adjacent protomers ${ }^{18}$. However, Rfc5 lacks residues critical for ATP hydrolysis and does not form a composite active site due to its position at the gap in the AAA+ tier. Mutational studies suggest that all four ATPase sites in RFC are required for normal RFC function ${ }^{18-24}$. Biochemical studies moreover suggest that ATP-binding to RFC promotes sequential PCNA binding, PCNA opening, and DNA binding by the RFC:PCNA complex, while ATP-hydrolysis induces the release of closed PCNA on DNA ${ }^{7,25-27}$. The structural basis for these ATP-driven events is not clear.

In previous structural studies, which lacked DNA, RFC adopts an inactive conformation in complex with a closed PCNA ring ${ }^{16,17}$. In these structures, PCNA contacts Rfc1, -4 , and -3 , but is freely suspended over Rfc2 and -5 . However, PCNA clamp opening is thought to involve interactions with all RFC subunits, which was suggested to force the closed planar PCNA ring into an open spiral conformation that matches the pitch of the spirally arranged RFC subunits 16. Such an out-of-plane clamp-opening mechanism is supported by molecular dynamics simulations of the PCNA ring and structural analyses of homologous clamp loader:clamp:DNA complexes from bacteriophage T4 and archaea ${ }^{28-30}$. In this model, PCNA will form a gap of 
$<10 \AA$, which would preclude the insertion of dsDNA. However, by analogy to the clamp loader complexes from Escherichia coli and bacteriophage T4, and in accordance with previous DNase footprinting and mutational analyses, RFC is thought to bind to the duplex region of 3' junctions inside its central channel, loading PCNA onto the adjacent double-stranded DNA 15,29,31,32. A "screw-cap model" that reconciles the narrow gap predicted for the open RFCPCNA complex with PCNA loading over dsDNA was proposed in which the open RFC-PCNA complex initially threads onto single-stranded DNA and subsequently slides towards the 3' junction for functional positioning ${ }^{16}$. Direct evidence for this model is currently lacking.

In addition to the $\mathrm{AAA}+$ and collar domains, Rfc1 contains unique $\mathrm{N}$ - and $\mathrm{C}$-terminal extensions that are absent in Rfc2-5. The C-terminus of Rfc1 contains the A' domain that is situated between the AAA+ domains of $\operatorname{Rfc} 1$ and $\operatorname{Rfc} 5{ }^{16,17}$. The structural organization of the Rfc1 N-terminal domain (NTD) in the context of the RFC complex is not known. It has been noted early on that a region of the Rfc1 NTD exhibits significant homology to domains found in prokaryotic DNA ligases and PARP1 ${ }^{33-35}$. Subsequent sequence analysis suggested that the Rfc1 NTD encompasses a single BRCT domain that forms a distinct BRCT subclass with single BRCT domains found in prokaryotic DNA ligases and PARP1 ${ }^{36}$. This prediction was confirmed by NMR analysis of the human Rfc1 BRCT domain ${ }^{37,38}$. Surprisingly, unlike tandem BRCT domains in eukaryotes that mediate phosphorylation-dependent protein interactions, the BRCT domain of Rfc1 was found to harbor a structure-specific DNA binding activity that has increased affinity for double-stranded DNA featuring a recessed and phosphorylated 5' end $34,35,38,39$.

The functional significance of the Rfc1 NTD has remained enigmatic. On the one hand, biochemical studies with truncated RFC complexes have demonstrated that the Rfc1 NTD is not essential for PCNA loading in vitro or for normal growth of budding yeast cells ${ }^{40-42}$. Moreover, RFC lacking the Rfc1 NTD was found to exhibit even greater activity in vitro than 
the full-length protein, which has been variably attributed to the greater protein stability or the loss of non-specific DNA binding activity of RFC lacking the Rfc1 NTD ${ }^{40-42}$. On the other hand, mutations in the Rfc1 NTD cause a cold-sensitive growth defect in budding yeast, while both truncation and mutation of the Rfc1 NTD increase sensitivity of budding yeast cells to the DNAdamaging agent methyl methanesulfonate (MMS) $40,43,44$. Truncation of the NTD in human RFC1 has also been associated with Hutchinson-Gilford progeria syndrome ${ }^{45}$. Combined with the high degree of conservation of the Rfc1 NTD across eukaryotes this data suggests that the Rfc1 NTD performs an important but undefined function ${ }^{46}$.

To address the mechanism of PCNA loading by RFC, we have reconstituted the budding yeast RFC:PCNA complex bound to a DNA substrate harboring a recessed 3' junction and examined its structure using cryogenic electron microscopy. Contrary to previous models, our data reveal that PCNA is opened in a largely planar fashion and that PCNA closing around DNA can occur in the absence of ATP hydrolysis. Moreover, by resolving multiple DNA-bound states of the RFC:PCNA complex we uncover the mechanism by which dsDNA is inserted into the central channel formed by RFC:PCNA. Finally, we resolve the DNA-bound structure of the $\mathrm{N}$-terminal BRCT domain in the context of RFC:PCNA:DNA complexes and demonstrate that this domain promotes PCNA loading at replication forks in vitro. Combined, our data suggest a novel and comprehensive model for the RFC-catalyzed loading of PCNA at 3' junctions that refutes previous models.

\section{Results:}

\section{Structure of RFC:PCNA in an active state}

We expressed and purified the full-length RFC complex composed of Rfc1-5 from S. cerevisiae cells, and trimeric PCNA and the Replication Factor A (RPA) complex from E. coli 
(Figure S1). The ATPase activity of purified RFC was stimulated by the presence of PCNA and DNA containing a 3' ss/dsDNA junction, indicating that the purified components are functional. RPA has been shown to promote the loading of PCNA by RFC specifically at 3' ss/dsDNA junctions ${ }^{13}$. Therefore, to resolve the structure of RFC actively loading PCNA onto DNA, we preincubated RPA with a DNA substrate containing a 20-base double-stranded segment and a 30-base 5' overhang, then assembled the full RFC:PCNA:DNA complex in the presence of saturating ATP $\gamma$ S, separated intact complexes by glycerol gradient centrifugation and collected cryogenic electron microscopic images (Figures S1 and S2). Image analysis revealed the presence of multiple distinct conformations of RFC:PCNA in the presence of a 3' ss/dsDNA junction at resolutions from 2.9 to $2.1 \AA$ (Figure $S 2$ and Table 1). In all of the conformations, RFC:PCNA is composed of three layers; a bottom layer comprised of the collar domains of the five RFC subunits, a middle layer of the AAA+ domains of the five RFC subunits and the Cterminal A' domain of Rfc1 and a top layer of the PCNA homotrimer (Figure 1A-C). The most notable difference between the three conformations is the arrangement of the PCNA ring (Figure 1D-F). In classes 1 and 2, PCNA is dilated in a planar fashion compared to crystal structures of trimeric PCNA ${ }^{2,3}$. In class 1, the space between PCNA protomers measures 22 $\AA$, which is wide enough to accommodate dsDNA. The gap in class 2 is much narrower and unable to accommodate dsDNA. In class 3, PCNA is fully closed and resembles structures of PCNA alone and in complex with RFC in an inactive state ${ }^{3,16}$. We will thus refer to class 1 as open, class 2 as intermediate and class 3 as closed.

\section{RFC stabilizes PCNA in an open state}

In the open state, the AAA+ domains of all five RFC subunits bind to PCNA (Figure 2A). Due to a mismatch between heteropentameric RFC and homotrimeric PCNA, there are several different types of interfaces between the subunits of RFC and PCNA. Rfc1, Rfc3 and Rfc5 bind 
in the hydrophobic grooves between the $\mathrm{N}$ - and $\mathrm{C}$-terminal domains of PCNA, $\mathrm{PCNA}_{\mathrm{B}}$, and PCNAc, respectively. Rfc4 and Rfc2 bind at the inter-subunit interfaces between $\mathrm{PCNA}_{\mathrm{A}}$ and $\mathrm{PCNA}_{\mathrm{B}}$, and PCNA $\mathrm{P}$ and PCNAC, respectively. The contacts established by Rfc1, Rfc3 and Rfc5 are larger and are comprised of both hydrophobic and hydrophilic interactions, while those established by Rfc4 and Rfc2 at the inter-subunit interfaces are smaller and largely hydrophilic (Figure 2A). Unique among the RFC subunits, Rfc1 forms a second interaction with PCNA via it's C-terminal A' domain, resulting in each PCNA protomer associating with two RFC subunits in the open state (Figure 2A). Together, these interactions stabilize PCNA in an open horseshoe-like shape that is patterned on the shape of the AAA+ and $A^{\prime}$ domains of RFC.

The planar orientation of PCNA relative to RFC in RFC:PCNA:DNA is distinct from the tilted orientation resolved for PCNA in structures of yeast and human RFC:PCNA in inactive, DNA-free states ${ }^{16,17}$ (Figure S3). Also different are the positions of the AAA+ domains of RFC. In the inactive structures, the AAA+ domains of RFC adopt a spiral configuration whereas the AAA+ domains are arranged in a largely planar fashion in RFC:PCNA bound to a 3' ss/ds DNA junction. The rearrangement of the AAA+ domains of RFC coincides with the conserved arginine finger from the neighboring subunits moving into direct contact with the bound ATP $\gamma$ S in Rfc1, Rfc4, Rfc3 and Rfc2 (Figure S3). Thus, the open state of RFC represents an active, pre-hydrolysis state of RFC in which PCNA is fully engaged.

Compared to the open state, the $\mathrm{PCNA}_{\mathrm{A}}$ and PCNAc protomers in the closed state rotate inwards as rigid bodies in a planar fashion by $15^{\circ}$ and $25^{\circ}$, respectively (Figure 2D). The inward movement of PCNAc disengages it from RFC. In absence of its interaction with PCNA, the PCNA-binding site of Rfc5 adopts an alternative conformation in which it instead binds to the N-terminus of Rfc2 (Figure S4). In the open state, the loop following the $\alpha 4$ helix, which we 
call the $\alpha 4$ loop that is comprised of residues $120-135$, extends up and inserts into the hydrophobic groove of PCNAc, establishing a large interface. In the closed state, the $\alpha 4$ loop bends down and is sandwiched between the AAA+ domain of Rfc5 and the N-terminus of Rfc2. The $\alpha 4$ loop of Rfc5 also undergoes a conformational change during the opening of the $9-1-1$ checkpoint clamp ${ }^{47}$. However, the conformations resolved in the presence of PCNA are distinct from those resolved in Rad24-RFC:9-1-1 as the $\alpha 4$ loop adopts a $\beta$-hairpin in the open Rad24-RFC:9-1-1 structure and is disordered in closed Rad24-RFC:9-1-1 (Figure S4). Thus, flexibility within its $\alpha 4$ loop allows Rfc5 to bind to two distinct DNA clamps - PCNA and 9-1-1 and stabilize them in their open states during loading.

While the inward movement of PCNAc results in its disengagement from RFC in the closed state, PCNA $A$ remains tightly associated with RFC despite also pivoting inwards (Figure 2B). The interface between $\mathrm{PCNA}_{A}$ and Rfc1 is unchanged in the closed state because Rfc1 also rotates compared to the open state (Figure 2C). The inward rotation of Rfc1 yields a $10 \AA$ movement of Rfc1-Phe405, which is inserted into the hydrophobic groove of PCNAA in both the open and closed states (Figure 2G). Indeed, Rfc1-Phe405 is inserted into the central groove of all known structures of RFC in complex with PCNA, indicating that it plays a central role in stabilizing the RFC:PCNA interaction (Figure S5).

In the intermediate state, PCNA adopts a conformation that is a mixture of protomers that adopt open-like and closed-like states. In the intermediate state, PCNAc disengages from RFC to adopt a closed-like conformation. In contrast, Rfc1 and PCNAA, which move together as a single unit in all of the structures, are only slightly rotated and adopt more open-like positions that prevent $\mathrm{PCNA}_{\mathrm{A}}$ from binding to $\mathrm{PCNA} \mathrm{A}_{\mathrm{C}}$ and closing of the ring (Figures $2 \mathrm{E}, \mathrm{F}$ and S5). Notably, the conformational changes in RFC in the closed and intermediate states do not arise from changes in nucleotide binding state. Densities corresponding to $\mathrm{Mg}^{2+}$-coordinated 
ATP $\gamma \mathrm{S}$ are resolved in the nucleotide-binding sites of $\mathrm{Rfc} 1, \mathrm{Rfc} 4, \mathrm{Rfc} 3$ and $\mathrm{Rfc} 2$ in the closed and intermediate maps as is an ADP in the nucleotide-binding site of the non-catalytic Rfc5 (Figure S3). Based on comparisons between the open, intermediate and closed states, we propose a two-step mechanism for the opening of PCNA when complexed with ATP-bound RFC. In the first step, Rfc1 and PCNAA together pivot outwards, separating PCNA from $\mathrm{PCNA}_{C}$ to form an intermediate state. In the absence of its interaction with PCNA $A$, PCNA can rotate freely and sample a range of conformations, including the open conformation where it binds to Rfc5. Once PCNAc contacts with Rfc5, the flexible $\alpha 4$ loop becomes ordered and binds in the hydrophobic groove of PCNAc, stabilizing PCNA in the open state.

\section{3'ss/dsDNA junctions bind to RFC:PCNA at multiple sites}

In the maps for all three states, densities were sufficiently well resolved for us to model a portion of the 3' ss/dsDNA junction. In the closed and intermediate states, the doublestranded region of the DNA is resolved extending from the bottom of the central chamber of RFC through to PCNA, while the 5' overhang on the template strand passes through the opening between the AAA+ and A' domains of Rfc1 (Figures 3A,B and S6). When bound at this position, which we call site 1 , the double-stranded region of the DNA in the central chamber is coordinated by Rfc1, Rfc4, Rfc3 and Rfc2. The backbone of the template strand binds to conserved isoleucine and arginine residues on helix $\alpha 5$ of $\operatorname{Rfc} 4, \operatorname{Rfc} 3$ and $\operatorname{Rfc} 2$ and to the side chains of Rfc1-Ser384 and Rfc1-Thr386, while the backbone of the primer strand binds to the side chain of Rfc5-Asn80 (Figure S6). The side chain of Rfc1-Arg434 is inserted into the minor groove and interacts with the bases from both strands. Despite the surface of PCNA containing an abundance of positively charged arginine and lysine residues, we do not observe any direct coordination of the dsDNA by PCNA. 
The coordination of the double-stranded region in site 1 by RFC guides the 3' ss/dsDNA junction to its binding site on the collar domain of Rfc1. Binding to RFC:PCNA partially melts the double stranded region of the DNA near the junction and we resolve density for unpaired bases on the primer strand that extend into an opening between the AAA+ domains of Rfc1 and Rfc4 that is continuous with the bulk solvent (Figures 3B and S6). The last paired base of the double-stranded region of the primer strand binds to the side chain of Trp638 of Rfc1, which serves a role analogous to the separation pin of helicases. The unpaired region of the primer strand is stabilized through both hydrophobic and polar interactions. The first unpaired base of the primer strand binds to Rfc1-Phe582, while the phosphate backbone of the second and third unpaired bases are coordinated by Rfc4-Arg272 and Rfc4-Lys275, respectively. Notably, additional density is resolved extending from the last modelled base of the primer strand that could not be modelled, indicating that the number of unpaired bases on the primer strand varies among the imaged particles (Figure S6). The 5' overhang of the template strand is guided through the opening between the AAA+ and $A^{\prime}$ domains of Rfc1 through polar and hydrophobic interactions with Rfc5-Asn103, Rfc1-Asn459, Rfc1-Pro461, Rfc1-Arg464, Rfc1Phe552, Rfc1-Arg632, Rfc1-GIn636, Rfc1-Phe666, Rfc1-Trp669 and Rfc1-Leu670 (Figure S6). In the open state, the 3' ss/dsDNA junction binds between the collar, AAA+ and A' domains of Rfc1, which we call site 2 (Figure 3C,D and S7). The 3' junction binds to a hydrophobic surface at the interface between the collar and A' domains of Rfc1 formed by Phe666, Trp669 and Leu670, with Phe666 serving as the separation pin for the template strand (Figure 3D). The unpaired bases on the $5^{\prime}$ overhang of the template strand bind to Asn459, Phe552 and Arg663 of Rfc1. While the precise positions of bases cannot be determined due to disorder, we can follow densities corresponding to a portion of the 5' overhang on the template strand in a $5 \AA$ low-pass filtered map as it wraps around the outside of the collar domain of Rfc1 and re-enters RFC through the opening between the AAA+ 
domains of Rfc1 and Rfc4 (Figure S7). There, several bases are again sufficiently ordered for modelling, and we observe that two of the bases occupy the same positions as the unpaired bases of the primer strand in the closed and intermediate states, indicating that it is a highaffinity binding site for ssDNA.

Eight base pairs of the double-stranded region of the DNA in site 2 are resolved in the density map, extending from the junction through the gap between the AAA+ and $A^{\prime}$ domains of Rfc1 towards the opening in the PCNA ring between PCNAA and PCNAc. The backbone of the template strand in site 2 is coordinated by Ser674, Lys675, Lys678 and Arg681 on the A' domain of Rfc1 (Figure 3D and S7). While the gap between PCNA and PCNAc is sufficiently wide to accommodate dsDNA, the minimum distance between the AAA+ and $A^{\prime}$ domains of Rfc1 is only $15 \AA$. For a DNA to bind at site 2, it must adopt a highly distorted conformation. Indeed, the fourth and fifth bases of the double-stranded region base stack on the opposing strand and do not adopt canonical Watson-Crick base pairing to enable the DNA to access to the narrow opening (Figure S7).

In several of the maps, in addition to the well resolved DNA densities at sites 1 or 2 , weak densities resembling a second segment of dsDNA are present between the AAA+ and collar domains of Rfc1. To better resolve these densities and potentially model a third DNA binding site, we assembled and collected cryo-EM images of RFC:PCNA in complex with a second DNA substrate that contains a longer, 30-base pair double-stranded DNA segment and a shorter, 10-base 5' overhang (Figure S8). Image analysis revealed open, closed and intermediate classes in the presence of the second DNA substrate at resolutions from 2.8 to 2.1 A, several of which contained densities for two 3' ss/dsDNA junctions (Figure S8 and Table 1). In the closed map, one of the DNA molecules occupies site 1 and binds to RFC in a nearly identical fashion as the first substrate does when bound in site 1 (Figure S9). The densities for the second DNA between AAA+ and collar domains of Rfc1 are much clearer than in the maps 
with the first DNA substrate and we were able to model to a second 3' ss/dsDNA junction at a position we call site 3 (Figures 3E,F, S8 and S9). When bound to site 3, the double-stranded region of the DNA is partially melted and extends from the junction in a direction perpendicular to the clamp/clamp loader axis (Figure 3E). Rfc1 binds to both strands at the junction, with Phe552 interacting with the last base of the primer strand in a manner analogous to separation pins in helicases, while His556 and Ile664 interact with the last base of the template strand (Figure 3F). Several bases of the 3' overhang of the primer strand are well resolved and bind to a hydrophobic region of Rfc1 formed by Phe552, Phe587, Phe666 and Leu670 that guides the overhang towards the central chamber of RFC. Extending away from the junction, both strands of the double-stranded region form contacts with residues on the outside of the AAA+ domain of Rfc1. The primer strand is coordinated through interactions with the backbone nitrogen of Arg477 and the side chains of Asn459, GIn474 and Arg477, while the template strand is coordinated by interactions with the backbone of Lys314 and Gly315 (Figure S9).

The ss/dsDNA junctions in nicked DNA bind to RFC:PCNA at multiple sites

The 3 ' end of the primer strand in site 3 is located immediately adjacent to the 5 ' phosphate of the last resolved base at the 5' end of the template strand in site 1. Together the DNA junctions in sites 1 and 3 thus resemble two segments of dsDNA connected by a short single-stranded segment. RFC has been demonstrated to load PCNA at DNA nicks in vitro, which is relevant for RFC-PCNA function in DNA repair ${ }^{10,11,48}$. We, therefore hypothesized that RFC can bind and load PCNA at nicked DNA by binding one of the double-stranded segments to site 1 and the second segment to site 3 . We confirmed that our purified RFC could indeed load PCNA on nicked plasmid DNA using a DNA pull-down assay (Figure S10). To visualize PCNA loading at DNA nicks, we assembled and collected cryo-EM images of RFC:PCNA in complex with a 50 base-pair nicked dsDNA substrate (Figure S11). Image analysis revealed 
open and closed states at resolutions from 2.4 to $2.7 \AA$. Among these classes were open and closed states in which the nicked DNA was coordinated with one double-stranded segment in the central chamber at site 1 and the second double-stranded segment between the AAA+ and collar domains at site 3 connected by a single-stranded region (Figure 3G,H). These doublestranded segments are coordinated by Rfc1 in an identical fashion to how it coordinates 3' ss/dsDNA junctions in sites 1 and 3 with Trp582 and Gln636 binding to the last paired bases of the double-stranded region of the nicked DNA that occupies site 1 and Phe552, His556 and Ile664 binding the last bases of the double-stranded region of the nicked DNA that occupies site 3 (Figure $3 \mathrm{H}$ ). Moreover, both double-stranded regions of nicked DNA are melted and the 3' and 5' overhangs bind to the same residues of RFC as do the 3' and 5' overhangs of the 3' ss/dsDNA junctions that bind to RFC at sites 1 and 3, respectively. The 5-base single-stranded segment of the nicked DNA connecting the two double-stranded regions is coordinated by interactions with Phe552, Phe587, Arg632, Phe666, Trp669 and Leu670. Thus, RFC employs the same mechanism to load PCNA onto nicked DNA and 3' ss/dsDNA junctions.

\section{The Rfc1 NTD facilitates PCNA loading at replication forks}

Adjacent to the double-stranded portion of DNA in site 3, we observed additional protein densities in a subset of the particles with the second substrate into which we built the Nterminal domain (NTD) of Rfc1, which is absent or poorly resolved in the maps determined with the first and third substrates (Figures 4A, S2, S8, S11 and S12). The Rfc1 NTD is composed of a BRCT domain and a long $\alpha$-helical linker that is connected to the $\mathrm{N}$-terminus of the AAA+ domain by a short, disordered loop (Figure 4A). When ordered, the NTD primarily associates with the core of Rfc1 through polar interactions between the $\alpha$-helical linker and the AAA+ domain of Rfc1. These interactions are quite weak as 3D classification revealed that even 
when ordered the NTD can adopt multiple configurations with respect to the AAA+ domain of Rfc1. In contrast, the connection between the BRCT domain and the $\alpha$-helical linker seems be more rigid as they slide along the surface of the AAA+ domain as a single unit (Figure S12).

The NTD possesses an extended electropositive surface into which the bind the 5' overhang of the template strand and the double-stranded region of the DNA as it is extends away from RFC (Figure S12). From the 3' junction binding site on the collar domain, the 5' overhang is guided to the BRCT domain where it binds to Thr166, Gly167, Arg174, Lys208 and Lys209 (Figure 4B). Thr166, Gly167, Arg174 and Lys 209, which are all universally conserved among Rfc1 orthologs, form a binding site for the 5' phosphate of the last modelled base. The phosphate binding site, which was initially predicted based on comparison of a solution structure of a fragment of the NTD of human Rfc1, is remarkably similar to the structure of the phospho-peptide binding site of the BRCT domain of BRCA1 ${ }^{49}$ (Figure S12).

Extending away from the 3' ss/dsDNA junction, both strands of the double-stranded section of the DNA in site 3 bind to the Rfc1 NTD (Figure 3C). Notably, only the template strand, which binds to the side chain of Arg245, binds to the positively charged side chains of the NTD (Figure 4C). The primer strand makes numerous, but weaker contacts with the side chains of Thr189, Ser191, Ser193, and Ser194 and the backbone of Lys190. Together the extensive interactions that the NTD forms with both the 5 ' overhang and the double-stranded region of the DNA rationalize the preference of the Rfc1 BRCT domain for binding doublestranded DNA featuring a recessed and phosphorylated 5' end ${ }^{34,35,38,39}$. The participation of residues in the $\alpha$-helical linker moreover rationalize why the DNA binding affinity of a fragment containing residues flanking the human Rfc1 BRCT domain was greatly enhanced compared to the BRCT domain by itself ${ }^{38}$. 
To further evaluate the role of the NTD in loading PCNA onto different DNA substrates, we purified an RFC variant that lacks Rfc1 residues 2-280 (RFC-1 $\Delta \mathrm{N}$; Figure $5 \mathrm{~A}$ ) and compared its activities to that of full-length RFC (RFC-WT). Like RFC-WT, the ATPase activity of RFC-1 $1 \mathrm{~N}$ is maximally stimulated in the presence of both DNA and PCNA (Figures S1 and S10), indicating that the catalytic activity of RFC-1 $\Delta \mathrm{N}$ is not grossly affected by the absence of the NTD. To directly observe PCNA loading onto DNA, we monitored the association of PCNA with nicked circular plasmid DNA immobilized via biotin-linkage on paramagnetic streptavidin beads (Figure S10). In this assay, the association of PCNA with DNA is dependent on both ATP (Figure S10) and RFC (Figure S10), demonstrating that it is due to bona fide PCNA loading onto DNA. Importantly, under these conditions, RFC-1 $\Delta \mathrm{N}$ did not exhibit a noticeable PCNA loading defect, indicating that the Rfc1 NTD is not essential for PCNA loading at DNA nicks, as has also been noted previously ${ }^{48}$. In fact, RFC-1 $\Delta \mathrm{N}$ appeared to be slightly more active for PCNA loading in this assay than RFC-WT. However, as noted before ${ }^{40}$, full-length RFC has a greater propensity to form aggregates compared to RFC variants lacking the Rfc1 NTD and we, therefore, attribute the reduced PCNA loading activity of full-length RFC in this assay to its potentially enhanced aggregation on the DNA beads.

To circumvent potential issues due to protein solubility we compared the PCNAdependent ATPase activities of RFC-WT and RFC-1 $\Delta \mathrm{N}$ in solution, under dilute conditions (10 nM RFC-WT or RFC-1 $\Delta \mathrm{N}$ ) and in the presence of limiting concentrations of a 3' ss/dsDNA junction substrate. In these conditions, RFC-WT consistently exhibited a greater ATPase activity over the range of DNA concentrations tested than RFC-1 $\Delta N$ (Figure $5 B$ ). This data suggests that the Rfc1 NTD indeed promotes PCNA loading at 3' ss/dsDNA junctions.

We reasoned that a defect in PCNA loading in the absence of the Rfc1 NTD may cause defects in lagging strand synthesis at replication forks as primer extension during Okazaki 
fragment synthesis is mediated by Pol $\delta$, which is strictly dependent on PCNA for processive DNA synthesis ${ }^{50}$. To test this hypothesis, we performed DNA replication reactions on ARScontaining circular plasmid DNA templates in vitro in the absence of RFC or in the presence of varying concentrations of RFC-WT or RFC-1 $1 \Delta \mathrm{N}$ using the reconstituted budding yeast DNA replication system ${ }^{51,52}$. To differentiate leading and lagging strand products, reactions were carried out in the absence of Cdc9 (DNA ligase 1) and Fen1, which prevents nascent strand ligation (Figure 5C,D). As we have shown previously ${ }^{51}$, in the absence of RFC, Okazaki fragments are synthesized by Pol $\alpha$ or Pol $\alpha$ and Pol $\varepsilon$, resulting in a globally reduced Okazaki fragment length. As expected, addition of RFC-WT at a concentration as low as $10 \mathrm{nM}$ resulted in a marked increase in Okazaki fragment length, consistent with Pol $\delta$ carrying out the bulk of primer extension on the lagging strand. In contrast, full Okazaki fragment extension required 4 - 8 times higher concentrations of RFC- $1 \Delta \mathrm{N}$, indicating impaired Pol $\delta$ activity due to a PCNA loading defect in the absence of the Rfc1 NTD. Moreover, we note that leading strands, which normally terminate at the point of fork convergence at the plasmid pole opposite the replication origin, on average reached greater than half-unit lengths in the absence of RFC or at low concentrations of RFC- $1 \Delta \mathrm{N}$. Since leading strand synthesis terminates at the 5 ' end of the lagging strand of an opposing fork ${ }^{53}$, this effect could further indicate a defect in lagging strand synthesis in the absence of full RFC activity, allowing leading strands to be synthesized significantly beyond the point of sister replisome convergence. Alternatively, since PCNA is known to stabilize Pol $\varepsilon$ on the leading strand ${ }^{54,55}$, the stochastic stalling or slowing of one of the two forks emanating from the replication origin may shift the site of termination beyond the midpoint between the two opposing forks. Irrespective, the data is consistent with the Rfc1 NTD promoting PCNA loading at replication forks. 
Since the strand-displacement activity of $\mathrm{Pol} \delta$, which is absent in Pol $\alpha$ and Pol $\varepsilon$, is essential for Okazaki fragment ligation ${ }^{51}$, defects in PCNA-dependent Pol $\delta$ activity are expected to result in a corresponding defect in Okazaki fragment ligation. To test this prediction, we carried out in vitro replication reactions in the presence of Cdc9 and Fen1 (Figure 5E-G). In the absence of RFC, nascent strand ligation is severely compromised, as evidenced by the absence of covalently-closed plasmid daughters, which can be detected as plasmid supercoils by native agarose gel analysis in the presence of ethidium-bromide (EtBr), and the presence of largely unligated nascent strands in the denaturing gel analysis. In contrast, as little as $5 \mathrm{nM}$ RFC-WT results in the formation of a significant fraction of covalently-closed plasmid daughters and the generation of full-length or near full-length nascent strands in the denaturing gel analysis, indicative of efficient nascent strand ligation. Conversely, native gel analysis of the replication products obtained after addition of $5-40 \mathrm{nM} R F C-1 \Delta \mathrm{N}$ results in a greatly reduced formation of covalently-closed plasmid daughters and a concomitant increase in nicked or gapped plasmid daughters, while a prominent smear of partially ligated nascent strands is evident across the same range of RFC- $1 \Delta \mathrm{N}$ concentrations by denaturing gel analysis. In summary, we conclude that the Rfc1 NTD mediates efficient PCNA loading at replication forks, which is required for normal Okazaki fragment synthesis and ligation.

\section{Discussion:}

In previous structural studies, the RFC:PCNA complex was captured in an inactive state off DNA ${ }^{16,17}$. Moreover, the flexible NTD of Rfc1 was deleted in those studies to improve protein yield and solubility, precluding its structural and functional characterization. Therefore, to advance our insight into the PCNA loading process we have reconstituted RFC:PCNA:DNA complexes bound to ATP $\gamma \mathrm{S}$ with full-length proteins and analyzed their structure using cryo- 
EM, which allowed us to capture RFC:PCNA in an active state. We observe that PCNA can adopt both open and closed conformations of the in the presence of RFC, DNA, and ATP $\gamma$. The open state is consistent with previous biochemical studies demonstrating that ATPbinding, but not ATP-hydrolysis, is required for PCNA opening 7,18,32,56,57. Unexpectedly, however, the PCNA ring opens in a largely planar fashion, generating a gap of $\sim 22 \AA$ that aligns with the gap in RFC between the AAA+ and $A^{\prime}$ domains of Rfc1 and is wide enough for the passage of dsDNA. The wide opening of PCNA during loading is similar to that which we observed for the 9-1-1 checkpoint clamp by Rad24-RFC and also consistent with MD simulations and biochemical FRET studies suggesting that PCNA opens a $\sim 30 \AA$ gap during loading $7,47,58,59$. However, the wide opening of PCNA refutes a previous model implicating the adoption of a right-handed spiral conformation with a $<10 \AA$ gap by PCNA that was inspired by the spiral configuration of the RFC AAA+ domains observed in the crystal structure of inactive RFC bound to PCNA and supported by MD simulations on the isolated PCNA clamp 16,28. A spiral configuration for PCNA may be more prevalent in the absence of DNA, as our data suggests that the planar RFC-PCNA conformation is stabilized by DNA, which we discuss below. In any case, the open PCNA state observed here is compatible with the direct loading of PCNA around dsDNA and obviates the need to invoke ssDNA threading for RFC:PCNA positioning at 3' ss/dsDNA junctions, which agrees with the ability of RFC to load PCNA at DNA structures lacking extensive stretches of ssDNA, such as DNA nicks.

Closure of the PCNA ring occurs through a two-step process involving release of one protomer from Rfc5 and the concerted inward movement of a second protomer together with Rfc1. Notably, ATP $\gamma$ S remains actively coordinated in all four RFC catalytic sites in the closed PCNA state, demonstrating that PCNA closure is not mechanistically coupled to ATP hydrolysis. Instead, the open and closed states of PCNA appear to exist in a dynamic 
equilibrium that is driven by the binding energies of RFC-PCNA contacts. As kinetic studies have suggested that ATP-hydrolysis precedes PCNA closure ${ }^{25,27}$, it is, therefore, possible that ATP hydrolysis will drive PCNA ring closure by disrupting the wide-planar conformation of RFC. In addition, analogously to Rad24-RFC, we anticipate that ATP-hydrolysis will induce PCNA release via an out-of-plane motion of $\mathrm{Rfc}^{47}$.

The Rfc2-5 core can associate with four distinct large subunits, respectively, to form RFC (RFC), which contains Rfc1, or three RFC-like complexes, Ctf18-RFC, Rad24-RFC and Elg1-RFC ${ }^{60}$. Each clamp loader complex performs specific functions in the cell that are associated with a distinct DNA substrate specificity. For example, RFC loads PCNA specifically at 3' ss/dsDNA junctions and DNA nicks ${ }^{10-13}$, Ctf18-RFC loads PCNA at 3' ss/dsDNA junctions but not at DNA nicks ${ }^{10,61}$, Rad24-RFC loads the 9-1-1 clamp at 5' ss/dsDNA junctions ${ }^{12,47,62}$, and Elg1-RFC appears to unload PCNA from dsDNA ${ }^{63}$. It is, therefore, likely that the DNA substrate specificity of clamp loaders is determined by DNAprotein contacts involving the large subunit. Consistent with this notion, we have recently found that the 5' ss/dsDNA junction is exclusively coordinated by Rad24 in Rad24-RFC ${ }^{47}$. Similarly, we find here that 3' ss/dsDNA junctions are exclusively coordinated by Rfc1 in RFC. However, unlike in Rad24-RFC:9-1-1, where the DNA binds to a single site, we observe that DNA binds to three distinct sites in RFC:PCNA. In each case, the 3' ss/dsDNA junctions are positioned at distinct sites along a hydrophobic ridge on the surface of the Rfc1 collar domain. Intriguingly, several base pairs of the 3' ss/dsDNA junction are melted at each of these sites, utilizing distinct separation pin residues. DNA junction unwinding by RFC has not been noted before and was also not observed in the Rad24-RFC:9-1-1:DNA complex ${ }^{47}$. Because melting is independent of ATP-hydrolysis, it does not involve a helicase mechanism. Instead, it appears to be promoted by hydrophobic binding forces between DNA bases and hydrophobic side chains on the surface of RFC. As described below, our data suggests that junction unwinding 
drives the insertion of the DNA duplex into the central RFC channel at an intermediate step. In this regard, junction unwinding may aid RFC:PCNA binding particularly at DNA nicks or short gaps by extending the ssDNA stretch in the template strand. Alternatively, limited junction unwinding may stabilize RFC at 3' primer ends on the lagging strand and thereby facilitate displacement of Pol $\alpha$ from primer ends to promote the handover to Pol $\delta$ 51,64,65.

DNA site 1 is located in the center of the clamp loader, analogous to the binding sites resolved in the structures of the bacteriophage T4 and E. coli clamp loaders ${ }^{29,31}$. In this site, the phosphate backbone of the DNA duplex template strand forms interactions with conserved residues on all five clamp loader subunits, including those in the Rfc2-5 core that coordinate the ssDNA in Rad24-RFC, explaining how the Rfc2-5 core can be adapted to bind either ssDNA or dsDNA ${ }^{47}$. Moreover, we find that RFC can bind dsDNA in its central channel because Rfc1 lacks the loop that protrudes from the AAA+ domain of Rad24 that occludes dsDNA from binding in the central channel of Rad24-RFC. In agreement with the notion that the PCNA clamp exists in a dynamic equilibrium between open and closed states prior to ATPhydrolysis, we observe both states when DNA is present in the central channel. DNA binding at site 1 thus represents the final stage of the DNA loading reaction.

The second site is located between the AAA+, collar and $A^{\prime}$ domains of Rfc1. When bound to this site, the double-stranded portion of the DNA extends between the AAA+ and $A^{\prime}$ domains of Rfc1 towards the opening in PCNA and thus can only be occupied in the open state. DNA site 2, therefore, corresponds to the DNA binding site at an intermediate state that precedes occupation of DNA site 1. Interestingly, the gap between the Rfc1 AAA+ and collar domains is not wide enough to accommodate normal B-form DNA, causing a significant distortion in the DNA duplex as it transits into the central RFC channel. The energetic penalty associated with the dsDNA distortion indicates that the gap between the AAA+, collar and $A^{\prime}$ domains of Rfc1 acts as a gate. Rotation of the DNA induced by the unwinding of the 3' 
ss/dsDNA junction at the base of the gate may help push the DNA through the constriction of the gate, aided by the conformational flexibility of the open interface between the AAA+, collar and $A^{\prime}$ domains.

The third binding site is similar to the DNA junction binding site resolved in Rad24RFC:9-1-1:DNA ${ }^{47}$, located between the AAA+ and collar domains of Rfc1 and with the doublestranded region of the DNA extending away from the clamp loader. Also contributing to the third binding site is the BRCT domain of Rfc1, which contacts the phosphate backbone of the double-stranded DNA. The BRCT domain is anchored on the side of the Rfc1 AAA+ domain via a long linker helix and bridging DNA contacts. The BRCT domain and linker helix appear to form a rigid unit that is flexibly tethered to the AAA+ domain via a short linker. The flexible association of the BRCT domain promotes its ability to explore the 3D space around RFC for DNA targets, thus promoting the recruitment of RFC to the DNA substrate. Accordingly, while not being essential for RFC:PCNA binding to DNA at non-limiting concentrations of RFC or DNA substrate, the Rfc1 NTD is expected to increase the efficiency of RFC:PCNA:DNA complex formation. Consistent with this notion, we observe that the Rfc1 NTD promotes Okazaki fragment synthesis at replication forks, which likely requires rapid turnover of RFC. In addition, we note that simultaneous coordination of the DNA substrate at multiple DNA binding sites distributed across the BRCT, AAA+ and collar domains of Rfc1 may help restrain the conformational flexibility of Rfc1 and thus promote the active and planar conformation of RFCPCNA, which in turn may underlie the observation that both DNA and PCNA stimulate ATP binding to RFC ${ }^{25-27}$. DNA site 3 thus appears to be involved in the early stages of RFC:PCNA:DNA complex formation.

Among several weak BRCT-DNA contacts, which may aid a role for the BRCT domain in DNA scanning and handover to DNA site 2, we also observe discrete hydrogen bonds between a highly conserved TG motif of the BRCT domain and a backbone phosphate in the 
template strand near the 3'ss/dsDNA junction. This interaction may be responsible for the increased affinity of the Rfc1 BRCT domain for 5'-phosphorylated dsDNA fragments noted previously ${ }^{38,39}$ and may help position and stabilize RFC at the junction. Notably, the interaction of the TG motif with the DNA phosphate backbone appears to be conserved in the homologous PARP1 BRCT domain ${ }^{66}$. As has been noted previously, residues corresponding to the Rfc1 TG motif in tandem BRCT domains mediate the interaction with the phosphate moiety of phospho-peptides, indicating that the phospho-peptide binding activity of tandem BRCT domains has evolved from the ancestral DNA binding activity of single BRCT domains 37,38 .

Our data suggest the following model for PCNA loading (Figure 6): RFC-PCNA initially adopts an inactive and partially engaged conformation that searches for a DNA substrate using the flexible NTD of Rfc1 to probe the surrounding space. Binding of DNA to the BRCT domain then promotes occupation of DNA site 3 and induction of an active planar RFC:PCNA conformation, which shifts the equilibrium between the closed to open states of PCNA towards the open state. Driven by binding energy, the DNA substrate is released from DNA site 3 and transferred to DNA site 2 to initiate insertion into the central RFC:PCNA channel. By sliding and rotating over the hydrophobic surface of the Rfc1 collar domain, the DNA shifts into position at DNA site 1. DNA binding to site 1 then promotes ATP hydrolysis by RFC, which promotes PCNA closure by disrupting the planar RFC:PCNA interaction surface and consequently the dynamic interaction of PCNA with Rfc2 and -5 in the open state. In addition, ATP-hydrolysis is expected to eject PCNA from RFC via an out-of-plane motion of Rfc1. Finally, inactivated RFC releases from the DNA and re-enters the cycle by recruiting PCNA free in solution.

In our model, a step-wise RFC-DNA binding mechanism mediates the loading of PCNA at 3' ss/dsDNA junctions. While not being essential for RFC-DNA binding, the Rfc1 NTD 
promotes the initial interaction of RFC with DNA. Based on the MMS sensitivity of yeast cells lacking the Rfc1 NTD ${ }^{40}$, which may indicate a defect in the post-replicative processing of nicks or gaps in the DNA, we had initially hypothesized that the NTD is specifically required for PCNA loading at DNA nicks. However, we are unable to detect a defect in PCNA loading at DNA nicks in vitro in the absence of the Rfc1 NTD, which is consistent with previous studies demonstrating the proficiency of RFC lacking the Rfc1 NTD in loading PCNA at DNA nicks during mismatch repair in vitro ${ }^{48,67}$. We, therefore, hypothesize that PCNA loading at DNA nicks follows the same step-wise DNA binding mechanism as at 3' ss/dsDNA junctions with a single-stranded 5' overhang. However, our data do not discriminate between a transition of the 3' double-stranded segment from site 3 to site 2 or its direct binding of the second doublestranded region in site 2 , while maintaining the interaction of the 5 ' segment in site 3 . Through either pathway, RFC-PCNA will be bound to both the 3' and 5' junction of a DNA nick, as observed in our structural analysis here. Future studies will be necessary to elucidate the precise conformational transitions that nicked and gapped DNA undergo to yield the doublybound, pre-ATP-hydrolysis states that we captured.

In conclusion, our study reveals the structural basis for the loading of PCNA at 3' ss/dsDNA junctions by RFC, providing a framework for future studies aimed at understanding the mechanism of PCNA loading and unloading by RFC-like complexes. 


\section{Methods:}

\section{Protein expression and purification}

PCNA, RFC, and RPA were purified as previously described (Devbhandari et al., 2017). RFC-1 $\Delta \mathrm{N}$ lacks Rfc1 residues 2-280 and was purified identically to wildtype RFC using strain YJC17 (MATa ade2-1 ura3-1 his3-11,15 trp1-1 leu2-3,112 can1-100 pep4::kanMX bar::hphNAT1 his3::Gal-Gal4 trp1::Gal-Rfc2-Rfc3 ura3::Gal-Rfc4-Rfc5 leu2::Gal-Rfc1- $\Delta M$ ).

\section{Preparation of 3'ss/dsDNA template}

Oligos were purchased from IDT. DR2630: 5'TTTTTTTTTTTTTTTTTTTTTTTTTTTTTTTTTTTTTTTTTTTTTTTTTTGCGAGGAAGGACTGA GCAGG-3', DR2631: 5'-CCTGCTCAGTCCTTCCTCGC-3', DR2667: 5'TTTTTTTTTTGCGAGGAAGGACTGAGCAGGCTTCCATACC-3', DR2668: 5'GGTATGGAAGCCTGCTCAGTCCTTCCTCGC-3'. DR2630 was PAGE purified prior to template generation. The DNA oligos were annealed by mixing to a final concentration of 10 $\mu \mathrm{M}$ in $10 \mathrm{mM}$ Tris, $\mathrm{pH} 7.5 / 50 \mathrm{mM} \mathrm{NaCl} / 1 \mathrm{mM}$ EDTA, heat denaturation at $95^{\circ} \mathrm{C}$, and gradually decreasing the temperature from $95^{\circ} \mathrm{C}$ to $10^{\circ} \mathrm{C}$ in steps of $1^{\circ} \mathrm{C}$ per minute using a thermocycler.

\section{$\underline{\text { ATPase Assays }}$}

Reactions were carried out in buffer containing 5 mM Hepes- $\mathrm{KOH}, \mathrm{pH} 7.6$ / 100 mM $\mathrm{KOAc} / 2 \mathrm{mM} \mathrm{Mg}(\mathrm{OAc})_{2}$ / 5\% glycerol / 0.02\% NP40S / 1 mM DTT. RFC or RFC-1 $\triangle \mathrm{N}, \mathrm{PCNA}$, and 3' ss/dsDNA substrate were included at $10 \mathrm{nM}, 100 \mathrm{nM}$, and $10 \mathrm{nM}$, respectively, as indicated. Reactions were incubated at $30^{\circ} \mathrm{C}$ and at the indicated times $2 \mu \mathrm{L}$ aliquots were spotted on TLC PEI Cellulose F (Millipore) to stop the reaction. The TLC plates were developed in 0.6 M sodium phosphate buffer, scanned on a Typhoon FLA 7000 phosphoimager, and ATP hydrolysis quantified using ImageJ. For DNA titration experiments, 3'ss/dsDNA template was included at $20 \mathrm{nM}, 5 \mathrm{nM}$, and $2.5 \mathrm{nM}$, as indicated; the reaction time was 5 minutes.

\section{Preparation of bead-bound DNA template}


$16 \mu \mathrm{g}$ of plasmid DNA (p1285, $8.6 \mathrm{kbp}$ ) were nicked with $\mathrm{Nb} . \mathrm{BbvCl}$ for 2 hours. Nicked DNA was phenol-chloroform extracted and subsequently biotinylated via UV crosslinking following manufacturer instructions (Vector Laboratories, SP-1020) with the following modifications: DNA was crosslinked at $365 \mathrm{~nm}$ for 20 seconds. 2 mg Dynabeads M-280 Streptavadin (11205D) were washed once in $400 \mu \mathrm{L}$ buffer containing $25 \mathrm{mM}$ Hepes-KOH pH 7.6 / $1 \mathrm{mM}$ EDTA / $2 \mathrm{M} \mathrm{NaCl}$ and resuspended in $400 \mu \mathrm{L}$ of the same buffer. The biotinylated DNA was added to the beads and incubated overnight at room temperature with rotation. Beads were washed twice with $400 \mu \mathrm{L}$ buffer containing 25 mM Hepes-KOH pH 7.6 / 1 mM EDTA / 1M KOAc and once with $400 \mu \mathrm{L}$ buffer containing $25 \mathrm{mM}$ Hepes-KOH pH 7.6 / 1 mM EDTA. Beads were resuspended in $200 \mu \mathrm{L}$ of $25 \mathrm{mM}$ Hepes-KOH pH 7.6 / $1 \mathrm{mM}$ EDTA and stored at $4^{\circ} \mathrm{C}$.

\section{PCNA Loading Assay}

Reactions were carried out in a $15 \mu \mathrm{L}$ volume in buffer containing $25 \mathrm{mM}$ Hepes-KOH $\mathrm{pH} 7.6$ / 100 mM KOAc / 5 mM Mg(OAc) / 5\% glycerol / 0.01\% NP40S / 1 mM DTT. $10 \mu \mathrm{L}$ of DNA beads were used per reaction. RFC-WT or RFC-1 $\Delta \mathrm{N}$ were used at concentrations as indicated, while PCNA was included at $120 \mathrm{nM} .1 \mu \mathrm{L}$ of the reaction was taken as an input fraction. Reactions were incubated at $30^{\circ} \mathrm{C}$ for 5 minutes and terminated by addition of $3 \mu \mathrm{L}$ 0.5 M EDTA, $\mathrm{pH}$ 8.0. Unbound fraction was separated from beads using a magnetic rack and discarded, beads were washed twice with $500 \mu \mathrm{L}$ buffer containing $25 \mathrm{mM}$ Hepes-KOH pH 7.6 / 300 mM KOAc / 5 mM Mg(OAc) 2 / 5\% glycerol / 0.02\% NP40S. Finally, beads were resuspended in $30 \mu \mathrm{L}$ 1x Laemmli buffer, boiled, and analyzed by SDS-PAGE. For Western blot analysis, membranes were probed with monoclonal anti-FLAG-HRP (Sigma) to detect Rfc1 or polyclonal anti-PCNA (871; Zhang et al., Nature, 2000).

\section{Preparation of samples for cryo-EM grids.}

All reactions were carried out at $30^{\circ} \mathrm{C}$. First, PCNA $(4.28 \mu \mathrm{M})$ and RFC $(2.5 \mu \mathrm{M})$ were mixed for 30 minutes in a $49 \mu \mathrm{L}$ reaction containing $25 \mathrm{mM}$ Hepes- $\mathrm{KOH}$ pH $7.6 / 300 \mathrm{mM}$ KOAc / 10\% glycerol / 1 mM DTT / 2.04 mM ATPS / 5.1 mM Mg(OAc)2. Subsequently, RPA $(30 \mathrm{nM})$ and 3'ss/dsDNA $(1.5 \mu \mathrm{M})$ were added to the reaction, the reaction volume was increased to a final volume of $100 \mu \mathrm{L}$ with reaction buffer $(25 \mathrm{mM}$ Hepes-KOH pH 7.6 / 300 
$\mathrm{mM} \mathrm{KOAc} \mathrm{/} 7$ mM Mg(OAc) 2 / 5\% glycerol / 0.02\% NP40S / 1 mM DTT) and incubation continued for $30 \mathrm{~min}$. The reaction was layered onto a $4 \mathrm{~mL} 10-35 \%$ glycerol gradient containing 25 mM Hepes-KOH pH 7.6 / 300 mM KOAc / 7 mM Mg(OAc) / 5\% glycerol / 0.02\% NP40S / 1 mM DTT. Gradients were centrifuged at 45,000 rpm for 6 hours at $4^{\circ} \mathrm{C}$ in a Thermo Scientific AH-650 swing bucket rotor. $200 \mu \mathrm{L}$ fractions were manually collected from the top of the gradient and $10 \mu \mathrm{L}$ of each fraction analyzed by SDS-PAGE stained with SilverQuest Staining Kit (Invitrogen). Another $10 \mu \mathrm{L}$ of each fraction were subjected to DNA quantification using a QuBit 3.0 Fluorometer with QuBit dsDNA HS Assay kit (Q32851). Peak fractions were pooled and dialyzed against buffer containing $25 \mathrm{mM}$ Hepes-KOH, pH 7.6 / $300 \mathrm{mM} \mathrm{KOAc} \mathrm{/} 7$ $\mathrm{mM} \mathrm{Mg}(\mathrm{OAc})_{2}$. Dialyzed sample was concentrated via centrifugation in Amicon Ultracel $30 \mathrm{~K}$ $0.5 \mathrm{~mL}$ filter units (UFC503096) and the protein concentration of the final sample determined by SDS-PAGE and Coomassie stain by comparing with a known protein standard.

\section{$\underline{\text { Replication assays }}$}

$4 \mathrm{nM}$ ARS1-containing circular plasmid template (p1017, 4.8 kbp) was incubated with $20 \mathrm{nM}$ ORC, $20 \mathrm{nM}$ Cdc6 and $60 \mathrm{nM}$ Cdt1 $\cdot$ Mcm2-7, in the presence of $100 \mathrm{mM} \mathrm{KOAc}, 25 \mathrm{mM}$ Hepes-KOH pH 7.5, 5\% glycerol, 2.5 mM DTT, $0.02 \%$ NP-40 and 5 nM ATP for 20 min at $30^{\circ} \mathrm{C}$. $150 \mathrm{nM}$ of DDK was added and incubation continued for $20 \mathrm{~min}$ at $30^{\circ} \mathrm{C}$. Subsequently, a mastermix of proteins was added to yield final concentrations of $0.2 \mu \mathrm{g} / \mu \mathrm{lBSA}, 20 \mathrm{nM}$ Sld3·7, 125 nM Cdc45, 80 nM each dNTP, 16 nM CDK (Clb5-Cdc28), 100 nM GINS, 30 nM Pol $\varepsilon, 30$ nM Dpb11, 30 nM Sld2, 120 nM RPA, 60 nM Pol $\alpha, 35$ nM Ctf4, 75 nM PCNA, 4 nM Pol א, 25 nM Csm3-Tof1, 25 nM Mrc1, 0.2 mM rNTP, 30 nM Top1, 30 nM Top2, 0.04 mg/ml creatin kinase, $16 \mathrm{mM}$ creatine phosphate and $5 \mu \mathrm{Ci} \alpha-\left[{ }^{32} \mathrm{P}\right]-\mathrm{dATP}$ was added to the reaction. The salt concentration was increased to $190 \mathrm{mM} \mathrm{KOAc}$ using a $3 \mathrm{M}$ KOAc stock solution, RFC or RFC- $1 \Delta \mathrm{N}$ was added at concentrations as indicated in the figure, and reactions were initiated by the addition of $14 \mathrm{nM} \mathrm{Mcm} 10$. Fen1 and Cdc9 were included at $15 \mathrm{nM}$ each, as indicated. Reactions were incubated at $30^{\circ} \mathrm{C}$ for 30 minutes and stopped by addition of $40 \mathrm{mM}$ EDTA, $1.6 \mathrm{U}$ Proteinase $\mathrm{K}$, and $0.25 \% \mathrm{SDS}$, followed by incubation for $30 \mathrm{~min}$ at $37^{\circ} \mathrm{C}$. DNA was isolated from the reactions by phenol/chloroform-extraction and filtration through Illustra MicroSpin G-25 spin columns (GE). 
For denaturing gels, $0.8 \%$ agarose gels were cast in buffer containing $30 \mathrm{mM} \mathrm{NaOH}$ and $2 \mathrm{mM}$ EDTA and $10 \mu \mathrm{L}$ aliquots of the reactions were fractionated at $45 \mathrm{~V}$ for 3 hours in 30 $\mathrm{mM} \mathrm{NaOH}$. Gels were neutralized and fixed in 5\% TCA. For native gels, $10 \mu \mathrm{L}$ aliquots of the reactions were fractionated on $0.8 \%$ agarose gels containing $0.6 \mathrm{mg} / \mathrm{ml} \mathrm{EtBr}$, at $50 \mathrm{~V}$ for 3 hours in TAE. Gels were and imaged using Typhoon FLA 7000.

Quantification and lane traces of gel images were performed using ImageJ. For figure $5 \mathrm{~F}$, intensities of nicked and supercoiled products were computed relative to total intensity in each lane. The ratio of supercoiled to nicked products was then tabulated in Graphpad Prism.

\section{$\underline{\text { Cryo-EM sample preparation and data acquisition }}$}

For RFC:PCNA in the complex with each of the three DNA substrates, $3.5 \mu \mathrm{L}$ of purified protein at a concentration of $0.22 \mathrm{mg} / \mathrm{mL}$ was applied to Graphene Oxide Au 400 mesh QUANTIFOIL R1.2/1.3 holey carbon grids (Quantifoil), and then plunged into liquid nitrogencooled liquid ethane with a FEI Vitrobot Mark IV (FEI Thermo Fisher). The sample was frozen at $4^{\circ} \mathrm{C}$ with $100 \%$ humidity, using blotting times between 30 and 60 s and a waiting time of 30 s. Grids were transferred to a $300 \mathrm{keV}$ FEI Titan Krios microscopy equipped with a K3 summit direct electron detector (Gatan). Images were recorded with SerialEM ${ }^{68}$ in super-resolution mode at 29,000x, corresponding to super-resolution pixel size of $0.413 \AA$. Dose rate was 15 electrons/pixel/s, and defocus range was -0.5 to $-2.0 \mu \mathrm{m}$. Images were recorded for $3 \mathrm{~s}$ with 0.05 s subframes (total 60 subframes), corresponding to a total dose of 66 electrons/ $\AA^{2}$.

\section{Cryo-EM processing}

60-frame super-resolution movies (0.413 $\AA$ /pixel) of RFC:PCNA in the complex with dsDNA were gain corrected, Fourier cropped by two (0.826 Å/pixel) and aligned using wholeframe and local motion correction algorithms by cryoSPARC v3.2.069. Blob-based autopicking in cryoSPARC was implemented to select initial particle images. Several rounds of twodimensional classification were performed and the best 2D classes were manually selected for 
the initial 3D model generation using the ab initio algorithm in cryoSPARC. False-positive selections and contaminants were excluded through iterative rounds of heterogeneous classification using the model generated from the ab initio algorithm, followed by particle polishing in Relion 3.1.2 $2^{70}$. The polished particles were then classified using three-dimensional classification in cryoSPARC v3.3.1 ${ }^{71}$. Once classification had converged, the final particle stacks were refined using non-uniform refinement in cryoSPARC v3.3.1 with local CTF estimation and higher order aberration correction. The reconstructions were further improved by employing density modification on the two unfiltered half-maps with a soft mask in Phenix ${ }^{72}$.

\section{Model building and refinement}

The structures of the yeast RFC-PCNA complex (PDB: 1SXJ) ${ }^{16}$ were manually docked into the closed structure of RFC:PCNA with DNA substrate 1 in UCSF Chimera ${ }^{73}$. The models were manually rebuilt to fit the density and sequence in COOT ${ }^{74}$. The models were initially refined in ISOLDE ${ }^{75}$ to correct geometric errors before several cycles of manual rebuilding in COOT and real space refinement in Phenix ${ }^{76}$ against the closed state map.

The refined closed state model was manually docked into the open and intermediate state maps determined with DNA substrate 1 and manually rebuilt to fit the density in COOT. The final models were subjected to real space refinement in Phenix against the open state map. For DNA substrates 2 and 3, the appropriate DNA substrate 1 model was docked into the density maps, rebuilt in COOT and refined using real space refinement in Phenix.

Figures were prepared using PyMol (www.pymol.org), APBS ${ }^{77}$, UCSF Chimera ${ }^{73}$ and UCSF ChimeraX78. 
Acknowledgments: We thank M de la Cruz at the MSKCC Richard Rifkind Center for cryoEM for assistance with data collection and the MSKCC HPC group for assistance with data processing. This work was supported by NIH-NCI Cancer Center Support Grant P30 CA008748 (D.R, R.K.H.), NIGMS R01-GM107239 and NIGMS R01-GM127428 (D.R.), and the Josie Robertson Investigators Program (R.K.H). M.S. is a Walter Benjamin Fellow of the Deutsche Forschungsgemeinschaft.

Author contributions: J.C.C., M.S., D.R. and R.K.H. conceptualized, performed and analyzed the experiments. J.C.C. purified all proteins. M.S. collected and processed cryo-EM data. M.S and R.K.H. built models. S.D. and C.K. performed the replication assays. All authors contributed to writing the paper.

Competing interests: Authors declare that they have no competing interests.

Data availability: Cryo-EM maps and atomic coordinates have been deposited with the EMDB and PDB under accession codes EMDB-XXXXX and PDB XXXX for RFC:PCNA:DNA1 in the open state, codes EMDB-XXXXX and PDB XXXX for RFC:PCNA:DNA1 in the intermediate state, codes EMDB-XXXXX and PDB XXXX for RFC: PCNA:DNA1 in the closed state, code EMDB-XXXXX for RFC:PCNA:DNA2 in the consensus open state, codes EMDB-XXXXX and PDB XXXX for RFC:PCNA:DNA2 in the open state with NTD, code EMDB-XXXXX for RFC:PCNA:DNA2 in the consensus closed state, codes EMDB-XXXXX and PDB XXXX for RFC:PCNA:DNA2 in the closed state with NTD, codes EMDB-XXXXX and PDB XXXX for RFC: PCNA:nDNA in the open state, and codes EMDB-XXXXX and PDB XXXX for RFC: PCNA:nDNA in the closed state. Source data are provided with this paper. 
bioRxiv preprint doi: https://doi.org/10.1101/2022.02.09.479782; this version posted February 9, 2022. The copyright holder for this preprint (which was not certified by peer review) is the author/funder, who has granted bioRxiv a license to display the preprint in perpetuity. It is made available under aCC-BY-NC-ND 4.0 International license. 


\section{References:}

1. Boehm, E.M., Gildenberg, M.S. \& Washington, M.T. The Many Roles of PCNA in Eukaryotic DNA Replication. Enzymes 39, 231-54 (2016).

2. Gulbis, J.M., Kelman, Z., Hurwitz, J., O'Donnell, M. \& Kuriyan, J. Structure of the C-terminal region of p21(WAF1/CIP1) complexed with human PCNA. Cell 87, 297-306 (1996).

3. Krishna, T.S., Kong, X.P., Gary, S., Burgers, P.M. \& Kuriyan, J. Crystal structure of the eukaryotic DNA polymerase processivity factor PCNA. Cell 79, 1233-43 (1994).

4. $\quad \mathrm{Li}, \mathrm{H}$., Zheng, F. \& O'Donnell, M. Water skating: How polymerase sliding clamps move on DNA. FEBS J 288, 7256-7262 (2021).

5. Binder, J.K. et al. Intrinsic stability and oligomerization dynamics of DNA processivity clamps. Nucleic Acids Res 42, 6476-86 (2014).

6. Yao, N. et al. Clamp loading, unloading and intrinsic stability of the PCNA, beta and gp45 sliding clamps of human, E. coli and T4 replicases. Genes Cells 1, 101-13 (1996).

7. $\quad$ Zhuang, Z., Yoder, B.L., Burgers, P.M. \& Benkovic, S.J. The structure of a ring-opened proliferating cell nuclear antigen-replication factor C complex revealed by fluorescence energy transfer. Proc Natl Acad Sci U S A 103, 254651 (2006).

8. Kelch, B.A. Review: The lord of the rings: Structure and mechanism of the sliding clamp loader. Biopolymers 105, 532-46 (2016).

9. Arbel, M., Choudhary, K., Tfilin, O. \& Kupiec, M. PCNA Loaders and Unloaders-One Ring That Rules Them All. Genes (Basel) 12(2021).

10. Bylund, G.O. \& Burgers, P.M. Replication protein A-directed unloading of PCNA by the Ctf18 cohesion establishment complex. Mol Cell Biol 25, 5445-55 (2005).

11. Cai, J. et al. Reconstitution of human replication factor $C$ from its five subunits in baculovirus-infected insect cells. Proc Natl Acad Sci U S A 93, 12896-901 (1996).

12. Ellison, V. \& Stillman, B. Biochemical characterization of DNA damage checkpoint complexes: clamp loader and clamp complexes with specificity for 5' recessed DNA. PLoS Biol 1, E33 (2003).

13. Hayner, J.N., Douma, L.G. \& Bloom, L.B. The interplay of primer-template DNA phosphorylation status and single-stranded DNA binding proteins in directing clamp loaders to the appropriate polarity of DNA. Nucleic Acids Res 42, 10655-67 (2014).

14. Lee, S.H., Kwong, A.D., Pan, Z.Q. \& Hurwitz, J. Studies on the activator 1 protein complex, an accessory factor for proliferating cell nuclear antigen-dependent DNA polymerase delta. J Biol Chem 266, 594-602 (1991).

15. Tsurimoto, T. \& Stillman, B. Replication factors required for SV40 DNA replication in vitro. I. DNA structurespecific recognition of a primer-template junction by eukaryotic DNA polymerases and their accessory proteins. $J$ Biol Chem 266, 1950-60 (1991).

16. Bowman, G.D., O'Donnell, M. \& Kuriyan, J. Structural analysis of a eukaryotic sliding DNA clamp-clamp loader complex. Nature 429, 724-30 (2004).

17. Gaubitz, C. et al. Structure of the human clamp loader reveals an autoinhibited conformation of a substrate-bound AAA+ switch. Proc Natl Acad Sci U S A 117, 23571-23580 (2020).

18. Johnson, A., Yao, N.Y., Bowman, G.D., Kuriyan, J. \& O'Donnell, M. The replication factor C clamp loader requires arginine finger sensors to drive DNA binding and proliferating cell nuclear antigen loading. $J$ Biol Chem 281, 35531-43 (2006).

19. Sakato, M., O'Donnell, M. \& Hingorani, M.M. A central swivel point in the RFC clamp loader controls PCNA opening and loading on DNA. J Mol Biol 416, 163-75 (2012).

20. Schmidt, S.L., Gomes, X.V. \& Burgers, P.M. ATP utilization by yeast replication factor C. III. The ATP-binding domains of Rfc2, Rfc3, and Rfc4 are essential for DNA recognition and clamp loading. J Biol Chem 276, 34784-91 (2001).

21. Schmidt, S.L., Pautz, A.L. \& Burgers, P.M. ATP utilization by yeast replication factor C. IV. RFC ATP-binding mutants show defects in DNA replication, DNA repair, and checkpoint regulation. $J$ Biol Chem 276, 34792-800 (2001).

22. Marzahn, M.R., Hayner, J.N., Finkelstein, J., O'Donnell, M. \& Bloom, L.B. The ATP sites of AAA+ clamp loaders work together as a switch to assemble clamps on DNA. J Biol Chem 289, 5537-48 (2014).

23. Cai, J. et al. ATP hydrolysis catalyzed by human replication factor $\mathrm{C}$ requires participation of multiple subunits. Proc Natl Acad Sci U S A 95, 11607-12 (1998).

24. Podust, V.N., Tiwari, N., Ott, R. \& Fanning, E. Functional interactions among the subunits of replication factor C potentiate and modulate its ATPase activity. J Biol Chem 273, 12935-42 (1998). 
25. Chen, S., Levin, M.K., Sakato, M., Zhou, Y. \& Hingorani, M.M. Mechanism of ATP-driven PCNA clamp loading by S. cerevisiae RFC. J Mol Biol 388, 431-42 (2009).

26. Gomes, X.V., Schmidt, S.L. \& Burgers, P.M. ATP utilization by yeast replication factor C. II. Multiple stepwise ATP binding events are required to load proliferating cell nuclear antigen onto primed DNA. J Biol Chem 276, 34776-83 (2001).

27. Sakato, M., Zhou, Y. \& Hingorani, M.M. ATP binding and hydrolysis-driven rate-determining events in the RFCcatalyzed PCNA clamp loading reaction. J Mol Biol 416, 176-91 (2012).

28. Kazmirski, S.L., Zhao, Y., Bowman, G.D., O'Donnell, M. \& Kuriyan, J. Out-of-plane motions in open sliding clamps: molecular dynamics simulations of eukaryotic and archaeal proliferating cell nuclear antigen. Proc Natl Acad Sci U S A 102, 13801-6 (2005).

29. Kelch, B.A., Makino, D.L., O'Donnell, M. \& Kuriyan, J. How a DNA polymerase clamp loader opens a sliding clamp. Science 334, 1675-80 (2011).

30. Miyata, T. et al. Open clamp structure in the clamp-loading complex visualized by electron microscopic image analysis. Proc Natl Acad Sci U S A 102, 13795-800 (2005).

31. Simonetta, K.R. et al. The mechanism of ATP-dependent primer-template recognition by a clamp loader complex. Cell 137, 659-71 (2009).

32. Yao, N.Y., Johnson, A., Bowman, G.D., Kuriyan, J. \& O'Donnell, M. Mechanism of proliferating cell nuclear antigen clamp opening by replication factor C. J Biol Chem 281, 17528-17539 (2006).

33. Bunz, F., Kobayashi, R. \& Stillman, B. cDNAs encoding the large subunit of human replication factor C. Proc Natl Acad Sci U S A 90, 11014-8 (1993).

34. Burbelo, P.D., Utani, A., Pan, Z.Q. \& Yamada, Y. Cloning of the large subunit of activator 1 (replication factor C) reveals homology with bacterial DNA ligases. Proc Natl Acad Sci U S A 90, 11543-7 (1993).

35. Fotedar, R. et al. A conserved domain of the large subunit of replication factor C binds PCNA and acts like a dominant negative inhibitor of DNA replication in mammalian cells. EMBO J 15, 4423-33 (1996).

36. Bork, P. et al. A superfamily of conserved domains in DNA damage-responsive cell cycle checkpoint proteins. FASEB J 11, 68-76 (1997).

37. Kobayashi, M., Ab, E., Bonvin, A. \& Siegal, G. Structure of the DNA-bound BRCA1 C-terminal region from human replication factor C p140 and model of the protein-DNA complex. J Biol Chem 285, 10087-10097 (2010).

38. Kobayashi, M., Figaroa, F., Meeuwenoord, N., Jansen, L.E. \& Siegal, G. Characterization of the DNA binding and structural properties of the BRCT region of human replication factor C p140 subunit. J Biol Chem 281, 4308-17 (2006).

39. Allen, B.L. et al. DNA recognition properties of the N-terminal DNA binding domain within the large subunit of replication factor C. Nucleic Acids Res 26, 3877-82 (1998).

40. Gomes, X.V., Gary, S.L. \& Burgers, P.M. Overproduction in Escherichia coli and characterization of yeast replication factor C lacking the ligase homology domain. J Biol Chem 275, 14541-9 (2000).

41. Uhlmann, F., Cai, J., Gibbs, E., O'Donnell, M. \& Hurwitz, J. Deletion analysis of the large subunit p140 in human replication factor $\mathrm{C}$ reveals regions required for complex formation and replication activities. $J$ Biol Chem $\mathbf{2 7 2}$, 10058-64 (1997).

42. Podust, V.N., Tiwari, N., Stephan, S. \& Fanning, E. Replication factor C disengages from proliferating cell nuclear antigen (PCNA) upon sliding clamp formation, and PCNA itself tethers DNA polymerase delta to DNA. $J$ Biol Chem 273, 31992-9 (1998).

43. McAlear, M.A., Tuffo, K.M. \& Holm, C. The large subunit of replication factor C $(\mathrm{Rfc} 1 \mathrm{p} / \mathrm{Cdc} 44 \mathrm{p})$ is required for DNA replication and DNA repair in Saccharomyces cerevisiae. Genetics 142, 65-78 (1996).

44. Xie, Y., Counter, C. \& Alani, E. Characterization of the repeat-tract instability and mutator phenotypes conferred by a Tn3 insertion in RFC1, the large subunit of the yeast clamp loader. Genetics 151, 499-509 (1999).

45. Tang, H., Hilton, B., Musich, P.R., Fang, D.Z. \& Zou, Y. Replication factor C1, the large subunit of replication factor C, is proteolytically truncated in Hutchinson-Gilford progeria syndrome. Aging Cell 11, 363-5 (2012).

46. Cullmann, G., Fien, K., Kobayashi, R. \& Stillman, B. Characterization of the five replication factor C genes of Saccharomyces cerevisiae. Mol Cell Biol 15, 4661-71 (1995).

47. Castaneda, J.C., Schrecker, M., Remus, D. \& Hite, R. Mechanisms of loading and release of the 9-1-1 checkpoint clamp. bioRxiv (2022).

48. Pluciennik, A. et al. PCNA function in the activation and strand direction of MutLalpha endonuclease in mismatch repair. Proc Natl Acad Sci U S A 107, 16066-71 (2010).

49. Clapperton, J.A. et al. Structure and mechanism of BRCA1 BRCT domain recognition of phosphorylated BACH1 with implications for cancer. Nature Structural \& Molecular Biology 11, 512-518 (2004).

50. Kunkel, T.A. \& Burgers, P.M.J. Arranging eukaryotic nuclear DNA polymerases for replication: Specific interactions with accessory proteins arrange Pols alpha, delta, and in the replisome for leading-strand and laggingstrand DNA replication. Bioessays 39(2017). 
51. Devbhandari, S., Jiang, J., Kumar, C., Whitehouse, I. \& Remus, D. Chromatin Constrains the Initiation and Elongation of DNA Replication. Mol Cell 65, 131-141 (2017).

52. Devbhandari, S. \& Remus, D. Rad53 limits CMG helicase uncoupling from DNA synthesis at replication forks. Nat Struct Mol Biol 27, 461-471 (2020).

53. Dewar, J.M. \& Walter, J.C. Mechanisms of DNA replication termination. Nat Rev Mol Cell Biol 18, 507-516 (2017).

54. Kumar, C., Batra, S., Griffith, J.D. \& Remus, D. The interplay of RNA:DNA hybrid structure and G-quadruplexes determines the outcome of R-loop-replisome collisions. Elife 10(2021).

55. Yeeles, J.T.P., Janska, A., Early, A. \& Diffley, J.F.X. How the Eukaryotic Replisome Achieves Rapid and Efficient DNA Replication. Mol Cell 65, 105-116 (2017).

56. Gomes, X.V. \& Burgers, P.M. ATP utilization by yeast replication factor C. I. ATP-mediated interaction with DNA and with proliferating cell nuclear antigen. J Biol Chem 276, 34768-75 (2001).

57. Hingorani, M.M. \& Coman, M.M. On the specificity of interaction between the Saccharomyces cerevisiae clamp loader replication factor C and primed DNA templates during DNA replication. J Biol Chem 277, 47213-24 (2002).

58. Adelman, J.L., Chodera, J.D., Kuo, I.F., Miller, T.F., 3rd \& Barsky, D. The mechanical properties of PCNA: implications for the loading and function of a DNA sliding clamp. Biophys $J$ 98, 3062-9 (2010).

59. Tainer, J.A., McCammon, J.A. \& Ivanov, I. Recognition of the ring-opened state of proliferating cell nuclear antigen by replication factor C promotes eukaryotic clamp-loading. J Am Chem Soc 132, 7372-8 (2010).

60. Lee, K.Y. \& Park, S.H. Eukaryotic clamp loaders and unloaders in the maintenance of genome stability. Exp Mol Med 52, 1948-1958 (2020).

61. Bermudez, V.P. et al. The alternative Ctf18-Dcc1-Ctf8-replication factor C complex required for sister chromatid cohesion loads proliferating cell nuclear antigen onto DNA. Proc Natl Acad Sci U S A 100, 10237-42 (2003).

62. Majka, J., Binz, S.K., Wold, M.S. \& Burgers, P.M. Replication protein A directs loading of the DNA damage checkpoint clamp to 5'-DNA junctions. J Biol Chem 281, 27855-61 (2006).

63. Kubota, T., Katou, Y., Nakato, R., Shirahige, K. \& Donaldson, A.D. Replication-Coupled PCNA Unloading by the Elg1 Complex Occurs Genome-wide and Requires Okazaki Fragment Ligation. Cell Rep 12, 774-87 (2015).

64. Georgescu, R.E. et al. Mechanism of asymmetric polymerase assembly at the eukaryotic replication fork. Nat Struct Mol Biol 21, 664-70 (2014).

65. Yuzhakov, A., Kelman, Z., Hurwitz, J. \& O'Donnell, M. Multiple competition reactions for RPA order the assembly of the DNA polymerase delta holoenzyme. EMBO J 18, 6189-99 (1999).

66. Rudolph, J. et al. The BRCT domain of PARP1 binds intact DNA and mediates intrastrand transfer. Mol Cell 81, 4994-5006 e5 (2021).

67. Dzantiev, L. et al. A defined human system that supports bidirectional mismatch-provoked excision. Mol Cell 15, 31-41 (2004).

68. Mastronarde, D.N. Automated electron microscope tomography using robust prediction of specimen movements. $J$ Struct Biol 152, 36-51 (2005).

69. Punjani, A., Rubinstein, J.L., Fleet, D.J. \& Brubaker, M.A. cryoSPARC: algorithms for rapid unsupervised cryo-EM structure determination. Nat Methods 14, 290-296 (2017).

70. Scheres, S.H. Processing of Structurally Heterogeneous Cryo-EM Data in RELION. Methods Enzymol 579, 125-57 (2016).

71. Punjani, A., Zhang, H. \& Fleet, D.J. Non-uniform refinement: adaptive regularization improves single-particle cryoEM reconstruction. Nat Methods 17, 1214-1221 (2020).

72. Terwilliger, T.C., Ludtke, S.J., Read, R.J., Adams, P.D. \& Afonine, P.V. Improvement of cryo-EM maps by density modification. Nat Methods 17, 923-927 (2020).

73. Pettersen, E.F. et al. UCSF Chimera--a visualization system for exploratory research and analysis. J Comput Chem 25, 1605-12 (2004).

74. Emsley, P., Lohkamp, B., Scott, W.G. \& Cowtan, K. Features and development of Coot. Acta Crystallogr D Biol Crystallogr 66, 486-501 (2010).

75. Croll, T.I. ISOLDE: a physically realistic environment for model building into low-resolution electron-density maps. Acta Crystallogr D Struct Biol 74, 519-530 (2018).

76. Liebschner, D. et al. Macromolecular structure determination using X-rays, neutrons and electrons: recent developments in Phenix. Acta Crystallogr D Struct Biol 75, 861-877 (2019).

77. Jurrus, E. et al. Improvements to the APBS biomolecular solvation software suite. Protein Science 27, 112-128 (2018).

78. Goddard, T.D. et al. UCSF ChimeraX: Meeting modern challenges in visualization and analysis. Protein Sci 27, 1425 (2018). 


\section{Figures}
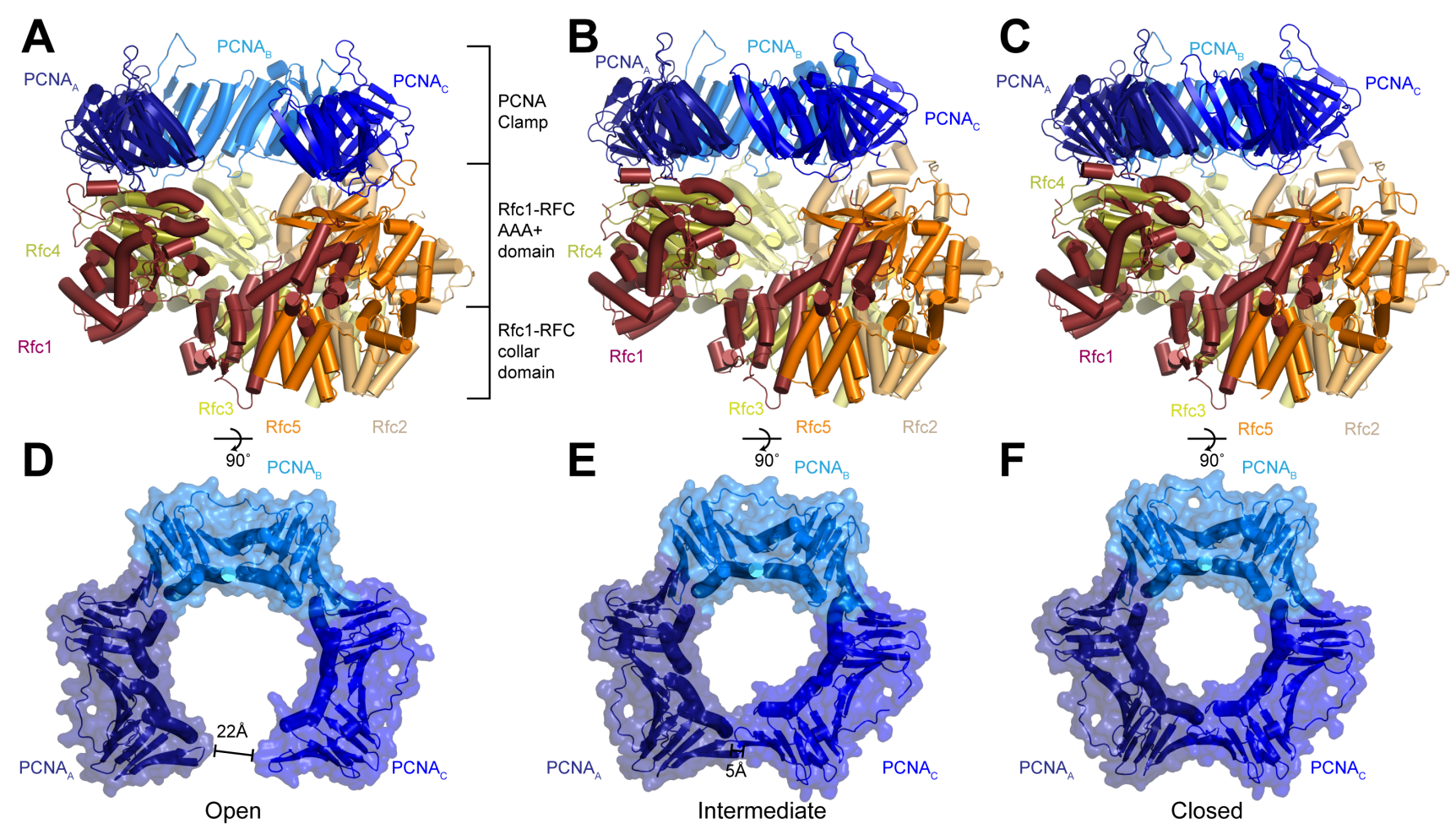

Figure 1. Structures of RFC in active states in complex with PCNA. A-C, Structures of RFC:PCNA in open (A), intermediate (B) and closed (C) states, colored by subunit. Rfc1 is maroon, Rfc4 is gold, Rfc3 is yellow, Rfc2 is sand, Rfc5 is orange and the PCNA protomers are blue. DNA is not shown for clarity. D-F, Open (D), intermediate (E), and closed (F) conformations of PCNA, colored by subunit. 

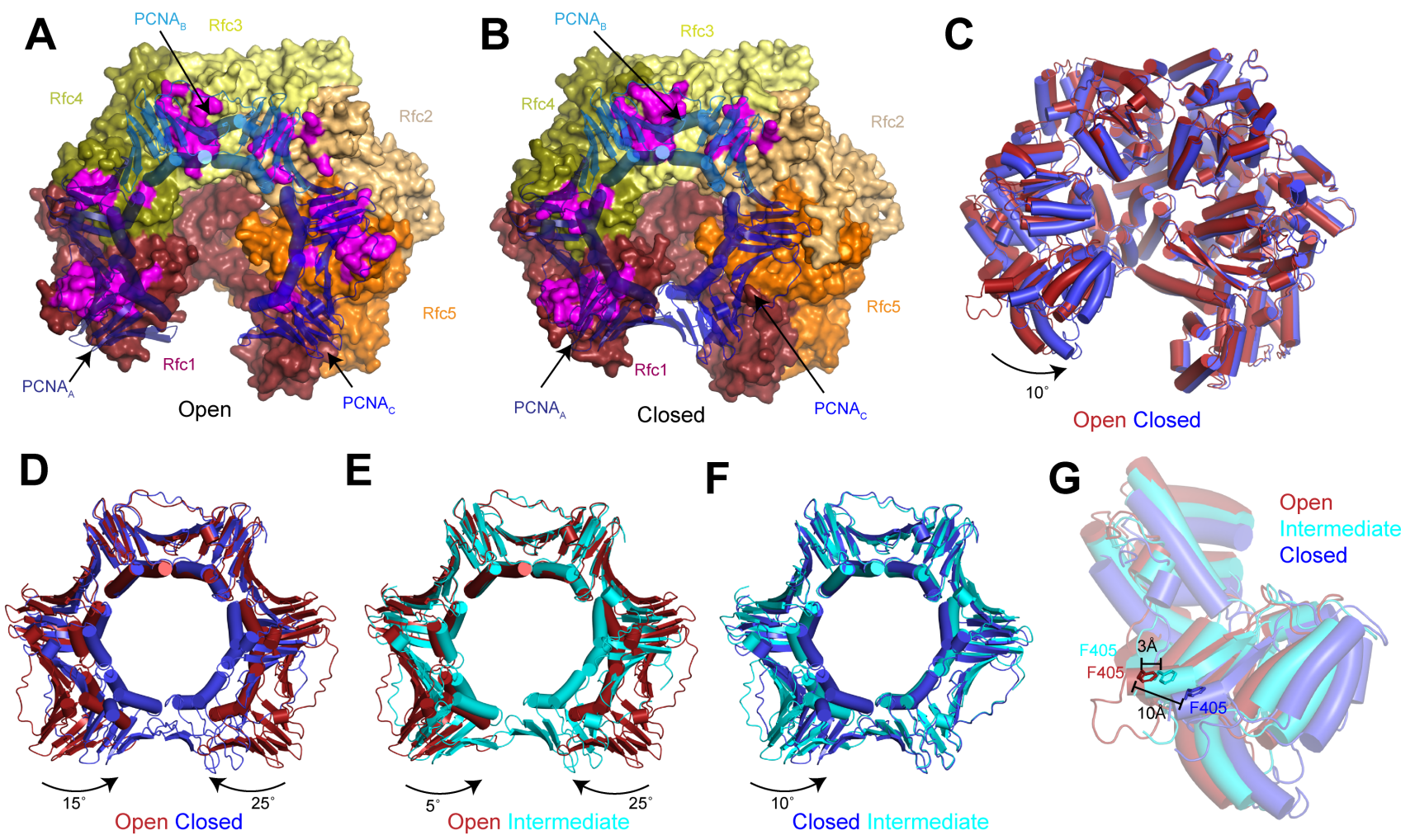

Figure 2. RFC stabilizes PCNA in the open conformation. A-B, Structures of RFC:PCNA in open (A) and closed (B) states. RFC is shown as a surface and PCNA is shown as a transparent cartoon. RFC and PCNA are colored by subunit with residues on RFC that interact with PCNA highlighted in magenta. C, Superposition of RFC in open (red) and closed (blue) states. Structures are aligned using Rfc3. D-F, Superpositions of PCNA between open and closed (D), open and intermediate $(E)$, and closed and intermediate states $(F)$. Structures are aligned using PCNA . Open PCNA is red, intermediate PCNA is cyan and closed PCNA is blue. G, RfC1 in open (red), intermediate (cyan) and closed (blue) states. Phe405 is shown as sticks. Structures are aligned using Rfc3. 
bioRxiv preprint doi: https://doi.org/10.1101/2022 02.09 .479782 ; this version posted February 9, 2022. The copyright holder for this preprint (which was not certified by peer review) is the author/funder, who has granted bioRxiv a license to display the preprint in perpetuity. It is made available under aCC-BY-NC-ND 4.0 International license.
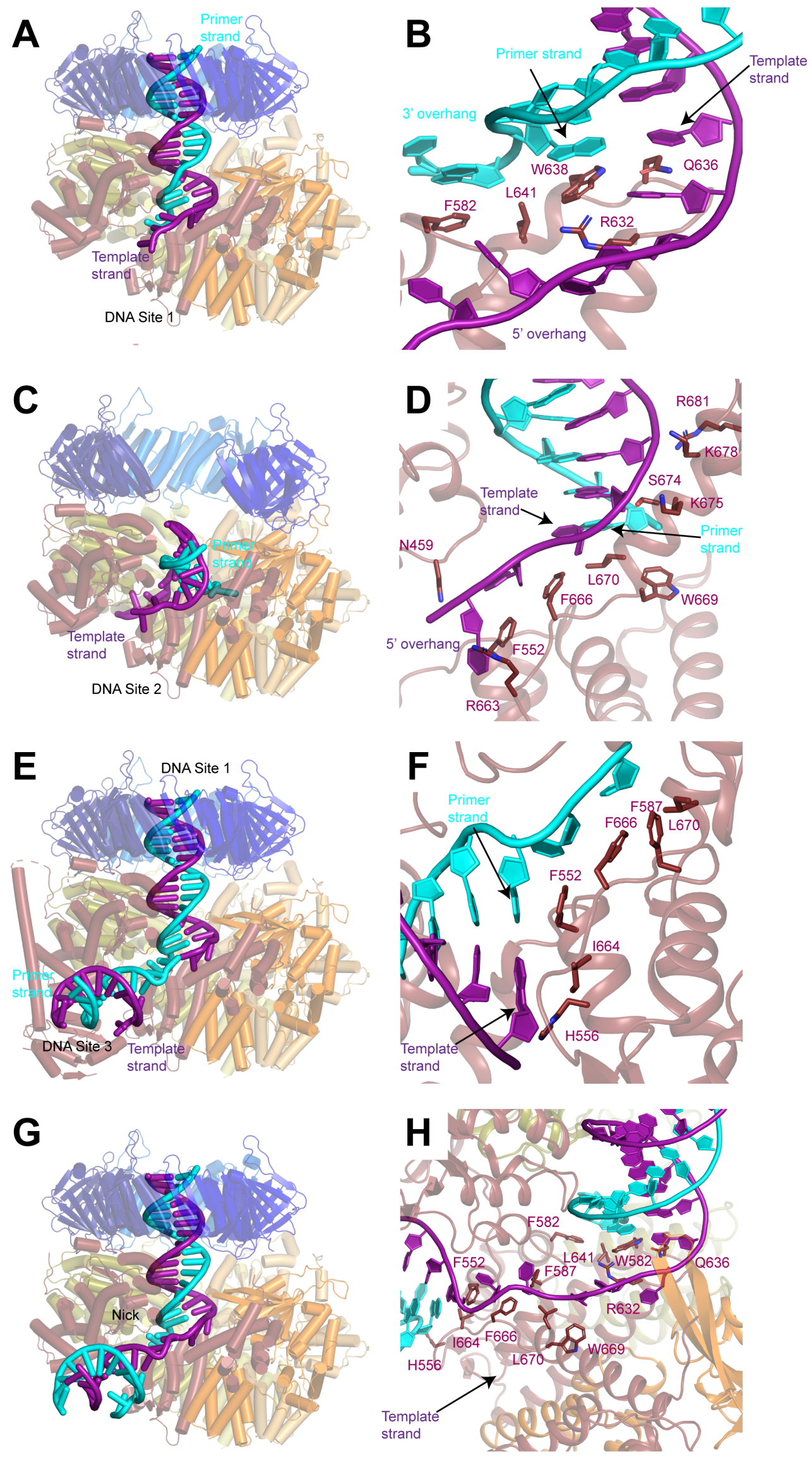
Figure 3. DNA binds to three sites in RFC. A, C, E, Structures of RFC:PCNA:DNA with 3' ss/dsDNA junctions occupying site 1 (A), site 2 (C) and sites 1 and $3(C)$. Labels are placed near the 5' ends of the template and primer strands. B, D, F, Coordination of 3' ss/dsDNA junctions by RFC at site $1(B)$, site $2(D)$ and site $3(F)$. Arrows point to the last paired bases on the template and primer strands. G, Structure of RFC in a closed state with a nicked dsDNA. $\mathbf{H}$, Coordination of nicked dsDNA by RFC at sites 1 and 3 . 

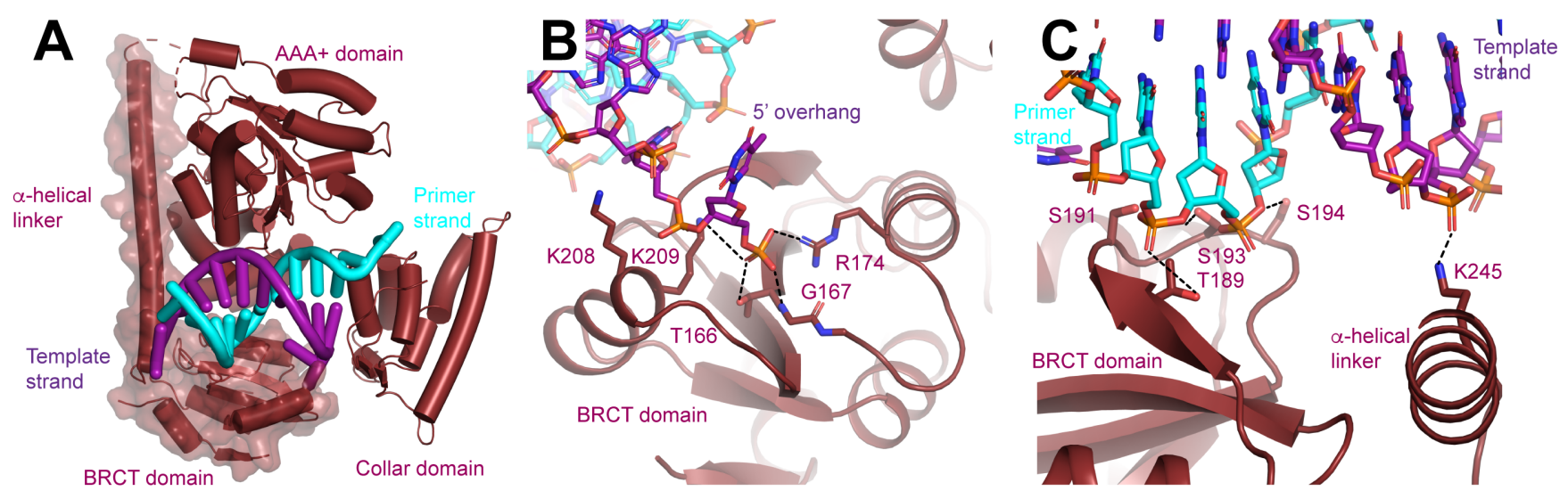

Figure 4. N-terminal domain of Rfc1. A, Structure of Rfc1 bound to a 3 ss/dsDNA junction in site 3. The NTD is shown as a surface and the A' domain is removed for clarity. The dashed line represents the disordered residues between the NTD and AAA+ domain of Rfc1. B, The BRCT domain binds the phosphate of the last ordered base on the 5' overhang of the template strand. C, The BRCT domain and the a-helical linker bind to both strands of a DNA in site 3. 
A

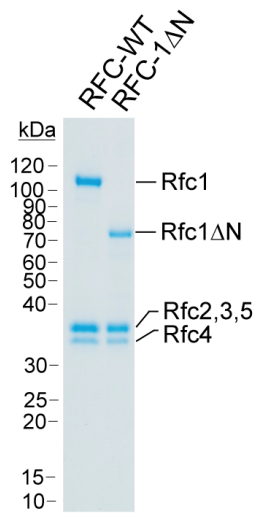

C

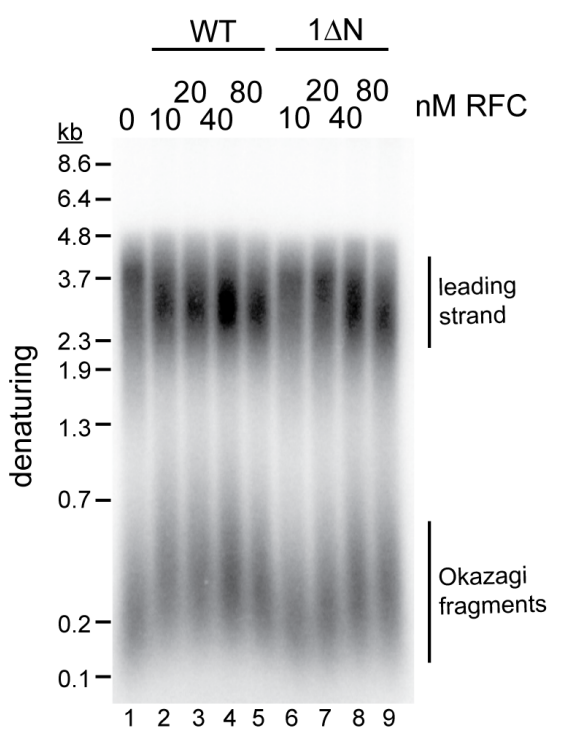

D

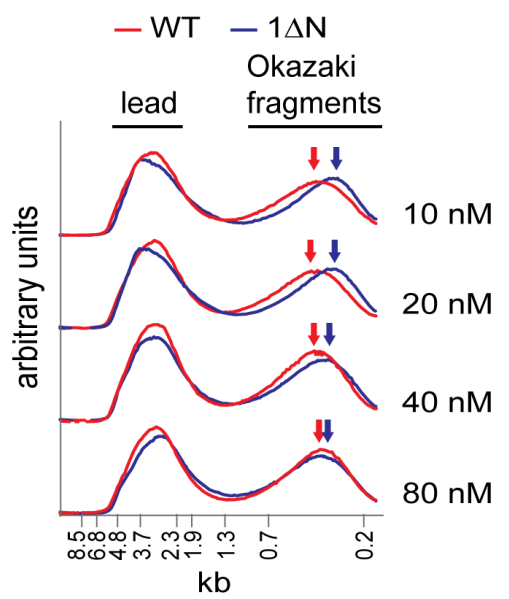

B

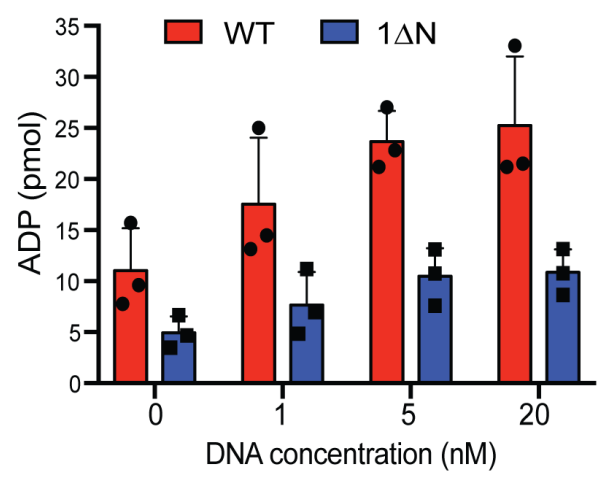

$\mathbf{E}$

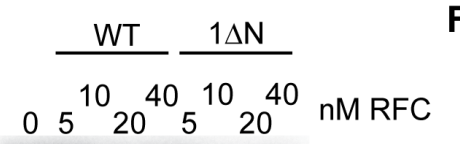

$\mathbf{F}$

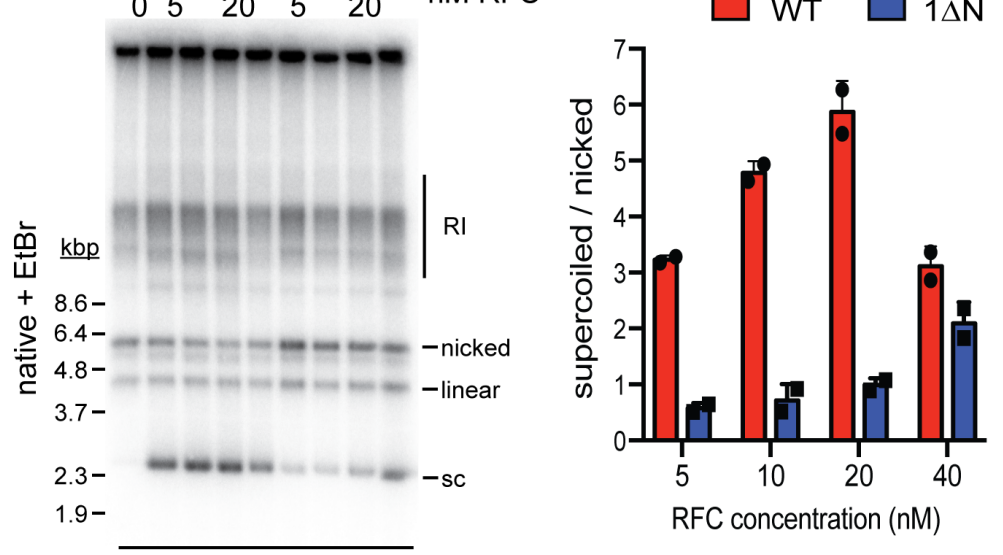

$\underline{\mathrm{kb}}$

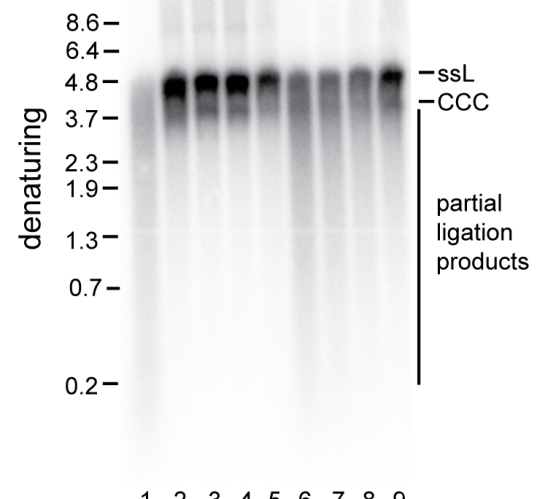

122344567789

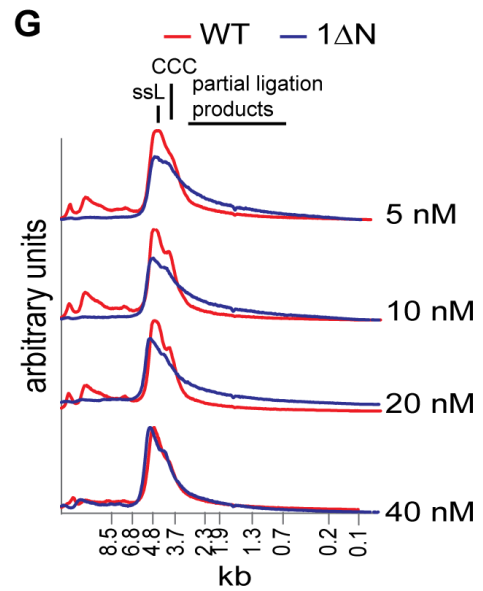

Figure 5. The Rfc1 N-terminal domain is required for efficient PCNA loading. A, Representative Coomassie-stained SDS-PAGE analysis of purified RFC-WT and RFC-1 $\Delta$ N. B, 
PCNA-dependent ATPase activity of RFC-WT (red bars) or RFC-1 $\Delta \mathrm{N}$ (blue bars) in the presence of varying DNA concentrations. Data are shown in triplicate. C, Denaturing gel analysis of replication products obtained in the absence (lane 1) or presence of variable concentrations of RFC-WT (lanes 2 - 5) or RFC-1 $\Delta \mathrm{N}$ (lanes 6 - 9). Reactions were performed in the absence of Cdc9 and Fen1. D, Lane traces of gel in panel C, lanes $2-5$ (red, RF-WT) and lanes 6 - 9 (blue, RFC-1 $\Delta N$ ). E, Replication products obtained in the presence of Cdc9 and Fen1 were analyzed by native agarose gel electrophoresis in the presence of ethidiumbromide (EtBr, top) or denaturing gel electrophoresis (bottom). RFC-WT (lanes 2- 5) or RFC$1 \Delta \mathrm{N}$ (lanes $6-9$ ) were included at concentrations indicated on top. Rl: Replication intermediates; sc: supercoiled; ssL: single-stranded linear; CCC: covalently closed circle. F, Ratio of supercoiled (fully ligated) to nicked (partially ligated) replication products observed by native agarose gel analysis in presence of $\mathrm{EtBr}$ as in panel $\mathrm{E}$. Data are shown in duplicate. G, Lane traces of replication products observed in denaturing gel analysis of panel E. Respective concentrations of RFC-WT (red) and RFC- $1 \Delta \mathrm{N}$ (blue) are indicated on the right. All assays were replicated at least two times. 

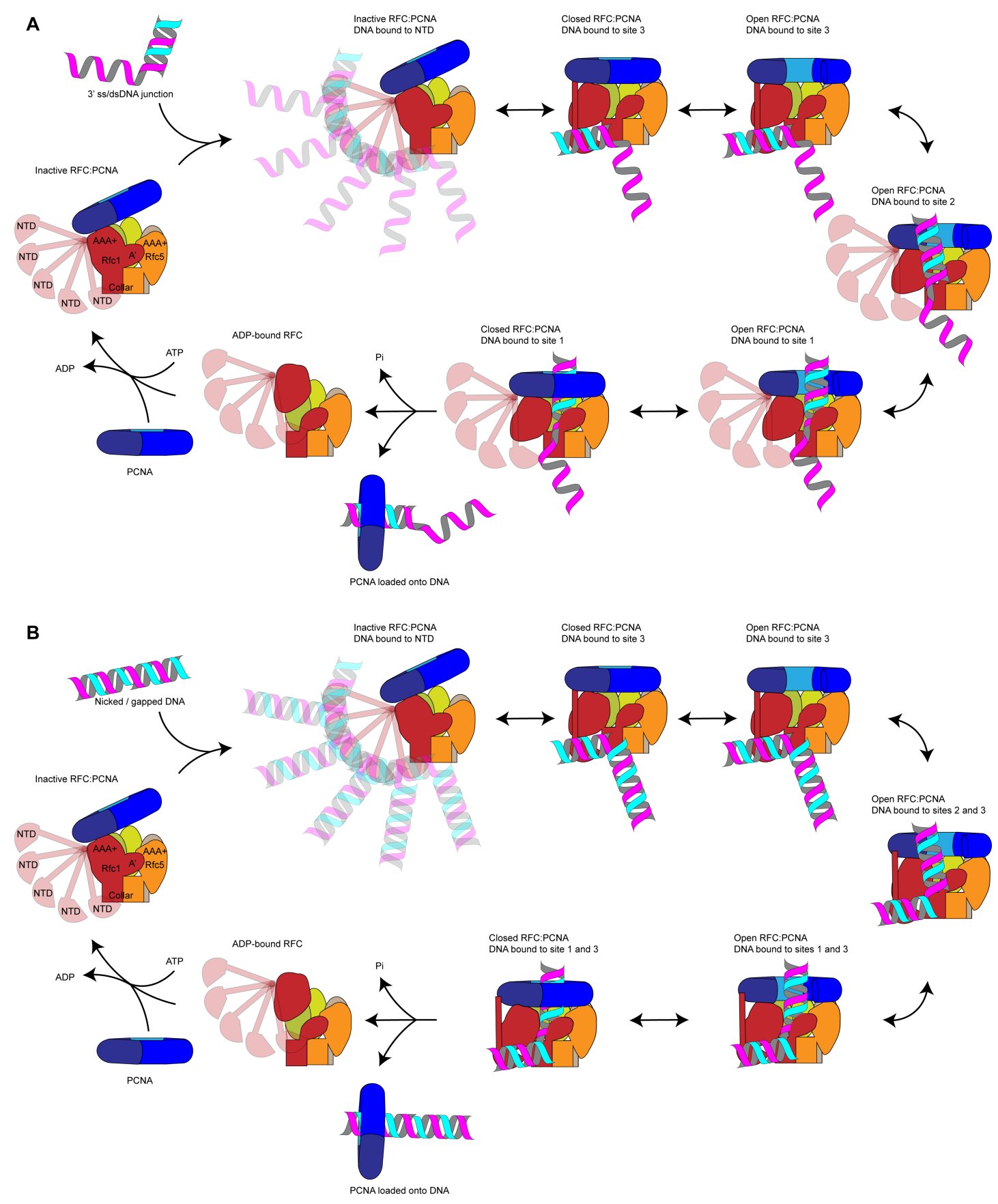

Figure 6. Loading for PCNA onto DNA by RFC. A, Model for PCNA loading onto 3' ss/dsDNA junctions. B, Model for PCNA loading onto nicked or gapped DNA. RFC and PCNA are colored by subunit as in Figure 1. 